\title{
35. NEOGENE PLANKTONIC FORAMINIFER MAGNETOBIOSTRATIGRAPHY OF THE SOUTHERN KERGUELEN PLATEAU (SITES 747, 748, AND 751) ${ }^{1}$
}

\author{
W. A. Berggren ${ }^{2}$
}

\begin{abstract}
With the exception of a brief ( 2 m.y.) late Miocene-early Pliocene hiatus, an essentially complete Neogene record was recovered on the Kerguelen Plateau in a calcareous biofacies. The stratigraphic distribution of about 30 taxa of Neogene planktonic foraminifers recovered at Sites 747, 748, and 751 (Central and Southern Kerguelen plateaus; approximately $54^{\circ}-58^{\circ} \mathrm{S}$ ) is recorded. Faunas are characterized by low diversity and high dominance and exhibit a gradual decline in species numbers (reflecting a concomitant increase in biosiliceous forms, particularly diatoms) from about 10 in the early Miocene to 5-8 in the middle Miocene, 3-4 in the late Miocene, to essentially a lone (Neogloboquadrina pachyderma) form in the Pliocene-Pleistocene. A provisional sevenfold biostratigraphic zonation has been formulated that, together with the recovery of a representative Neogene magnetostratigraphic record, may ultimately lead to a correlation with low-latitude magnetobiostratigraphies. The initial appearance of Neogloboquadrina pachyderma is associated with magnetic polarity Chron (MPC) 4 ( $~ 7 \mathrm{Ma}$ ) and MPC $4 \mathrm{~A}(>8 \mathrm{Ma})$ at Sites 747 and 751 , respectively.
\end{abstract}

\section{INTRODUCTION}

Ocean Drilling Program (ODP) Leg 120 drilled five sites in a transect spanning about $4^{\circ}$ latitude on the Central and Southern Kerguelen plateaus. The Neogene record of planktonic foraminifers is reported from Sites 747, 748, and 751 (see Fig. 1 and Table 1).

With the exception of several brief hiatuses, an essentially complete Neogene section was recovered on the Kerguelen Plateau in a calcareous biofacies. Although biosiliceous elements exhibit a trend toward increasing dominance during the Neogene, planktonic foraminifers are present throughout the sequence. Faunas exhibit features typical of high-latitude cool waters: low diversity and high dominance. Fluctuating abundance patterns, particularly in the late Neogene, reflect the repeated passage of the Polar Front across this region.

A provisional biostratigraphic scheme is developed for the Neogene of the Central and Southern Kerguelen plateaus, which, together with the recovery of a representative Neogene magnetostratigraphic record, may ultimately lead to a correlation with low-latitude magnetobiostratigraphies. The depths of Neogene magnetic polarity chronozones (MPC) identified in Sites 747, 748, and 751 are listed in Table 2; the stratigraphic positions of various biostratigraphic datum levels and chronostratigraphic boundaries are presented in a series of tables (3-8) that accompany site discussions.

\section{MATERIAL AND METHODS}

The samples discussed in this paper were collected and prepared aboard the JOIDES Resolution. Approximately one $\left(20 \mathrm{~cm}^{3}\right)$ sample per section (less frequently, one sample every second section) plus the core-catcher (CC) sample (which was examined on the ship) were examined. Samples were prepared by washing in warm water and wet sieving over a $63-\mu \mathrm{m}$ screen and dried in an oven at $<50^{\circ} \mathrm{C}$. The faunal composition

\footnotetext{
${ }^{1}$ Wise, S. W., Jr., Schlich, R., et al., 1992. Proc. ODP, Sci, Results, 120: College Station, TX (Ocean Drilling Program).

2 Department of Geology and Geophysics, Woods Hole Oceanographic Institution, Woods Hole, MA 02543, U.S.A., and Department of Geology, Brown University, Providence, RI 02912, U.S.A.
}

is reported here in semiquantitative terms based on an assessment of forms observed in a random sample of 200-400 specimens from the $>63-\mu \mathrm{m}$ size fraction. Abundances are recorded on the accompanying charts (Appendixes $\mathrm{A}-\mathrm{C}$, in back pocket) in terms of relative abundance; thus, abundant $(\mathrm{A})=>25 \%$, common $(\mathrm{C})=16 \%-24 \%)$, frequent $(\mathrm{F})=$ $6 \%-15 \%$, and rare $(\mathrm{R})=1 \%-5 \%$.

The generic classification used in this study follows, to a large extent, that of Loeblich and Tappan (1988). Species identifications relied heavily on Jenkins $(1966,1971)$, Berggren (1977), and Kennett and Srinivasan (1983). Taxonomic notes are provided to clarify concepts and usage where relevant.

\section{BIOSTRATIGRAPHY}

Neogene planktonic foraminifers were recovered at Sites 747,748 , and 751 . Faunas are characterized by low diversity and high dominance, a characteristic feature of high-latitude, cool-water assemblages. Neogene planktonic foraminifer occurrences are presented in a series of charts (Appendixes $\mathrm{A}-\mathrm{C}$, in back pocket), and the ranges of stratigraphically useful or otherwise distinctive forms are presented in a series of range charts that accompany the text (on microfiche in back pocket).

A variety of zonal schemes have been developed for the Austral mid- to high-latitude Neogene (see summary in Jenkins, 1975; Jenkins and Srinivasan, 1986; Berggren et al., 1983). A zonal biostratigraphy for the mid-latitude South Atlantic Neogene was formulated by Berggren (1977; see also Berggren et al., 1983). None of these zonal systems was fully applicable to the Neogene record of the Kerguelen Plateau. Elements of the Globorotalia miozea lineage figured prominently in the mid- to upper Neogene part of the zonal stratigraphies of these previously formulated schemes, but these forms ( $G$. conoidea $=G$. miotumida, $G$. conomiozea, $G$. sphericomiozea, G. puncticulata, and $G$. inflata) are essentially absent (or extremely rare and of sporadic occurrence only) in the Neogene of the Kerguelen Plateau. On the other hand, elements of the early part of the $G$. miozea group were common during the early Neogene and had a distribution similar to elsewhere in Austral and South Atlantic regions. In the mid to late Neogene, one or two taxa of globigerinids, 


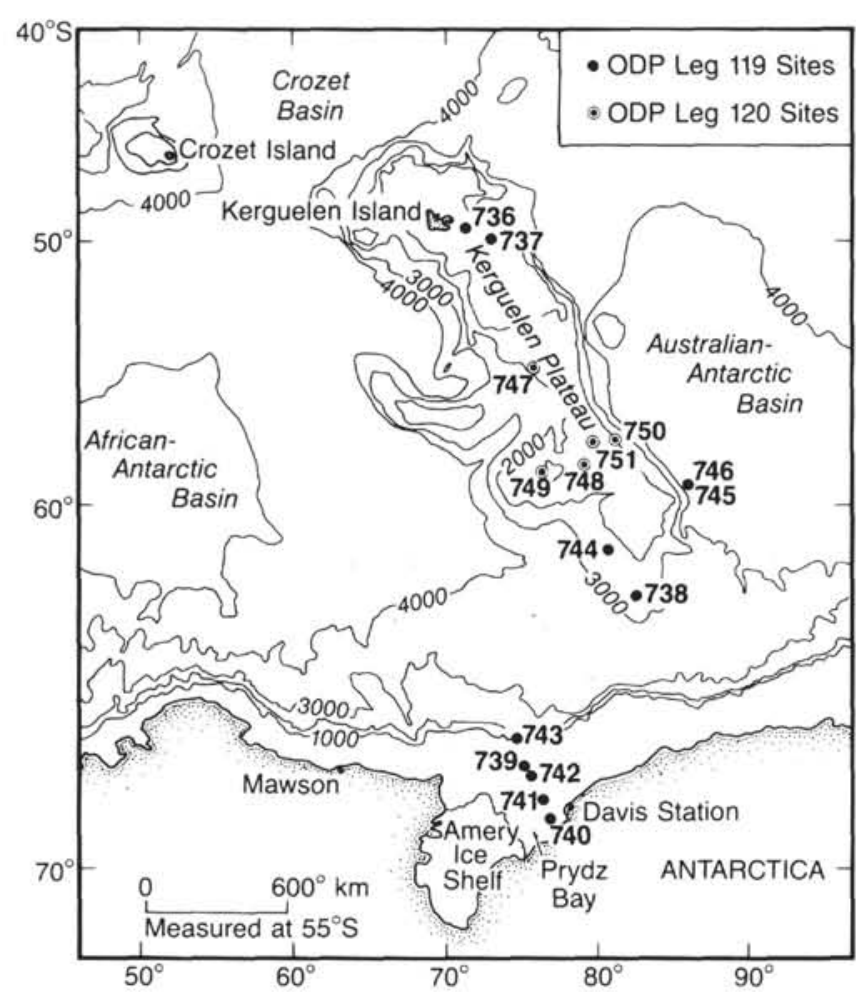

Figure 1. Map showing locations of Leg 120 sites on Kerguelen Plateau.

neogloboquadrinids, and globorotaliids were the dominant faunal elements reflecting the accelerating cooling and isolation of the (sub) Antarctic region from the central circulation system. We used the ranges of these forms to develop a zonal stratigraphy for this region.

I have formulated a provisional seven-fold zonal scheme of the Neogene that will be tested against other distribution patterns at high southern latitudes in the hope of ultimately developing a unified zonal scheme representative of, and applicable to, the circum-antarctic Neogene.

The notation "NK" in the zone system below stands for Neogene Kerguelen.

\section{ZONAL DEFINITIONS}

Globigerina brazieri (NK1) Partial Range Zone

Definition. Partial range of the nominate taxon from the last occurrence (LO) of Globigerina euapertura (base) to the first occurrence (FO) of Paragloborotalia incognita (top).

Remarks. This zone is characterized by an assemblage of globigerinids (including a large, robust, evolute form referred here to as Globigerina cf. labiacrassata), catapsydracids $(G$. unicavus $-G$. dissimilis), and Globorotaloides suteri. The last occurrence (LO) of $G$. euapertura has been found at Sites 747 and 748 to correspond closely to the Oligocene/Miocene boundary (as denoted by Subchron $\mathrm{C} 6 \mathrm{CN}$ ); thus, the base of this zone is considered to denote the (approximate) base of the Miocene.

\section{Paragloborotalia incognita (NK2) Partial Range Zone}

Definition. Partial range of the nominate taxon between its initial appearance (base) and the initial appearance of Globorotalia praescitula (top).

Remarks. Paragloborotalia incognita appears in the lower Miocene assemblages of the Kerguelen Plateau (MPC 6A) and apparently slightly higher than at Site 516 in the South Atlantic (MPC 6B) (Berggren et al., 1983). This taxon was used (together with its five-chambered "cousin," $P$. semivera) as a zonal marker for a biostratigraphic interval between the LO of Globorotalia kugleri (base) and the FO of G. praescitula (top).
Globorotalia kugleri does not occur in Kerguelen in lower Miocene assemblages (attesting to the significantly colder conditions in the early Miocene at Kerguelen compared with the Rio Grande Rise), but the extent and duration of Zone NK2 (Kerguelen) and M2 (South Atlantic) is essentially identical.

\section{Globorotalia praescitula (NK3) Partial Range Zone}

Definition. Partial range of the nominate taxon from its initial appearance (base) to the initial appearance of Globorotalia miozea (top).

Remarks. The FO of Globorotalia praescitula has been found to correspond to MPC 5E at Sites 747 and 751, which is apparently somewhat earlier than its recorded FO at Site 516 in association with MPC 5D (although this may also reflect problems with the interpretation of the paleomagnetic record). The total range of the morphologically similar forms $G$. zealandica and $G$. pseudomiozea was used to denote the early Miocene M3 Zone at the Rio Grande Rise (Berggren et al., 1983). These two forms are combined here as $G$. zealandica, which has been found to have a somewhat sporadic and irregular distribution pattern on the Kerguelen Plateau. At Site $747 \mathrm{G}$. zealandica ranges into the middle Miocene to a level correlative with MPC 5AD. As defined here, the G. praescitula Zone is correlative with Zone M3 of the South Atlantic (Berggren et al., 1983).

\section{Globorotalia miozea (NK4) Partial Range Zone}

Definition. Partial range of the nominate taxon from its initial occurrence (base) to the initial occurrence of Neogloboquadrina nympha (top).

Remarks. Globorotalia miozea appeared in late early Miocene faunal assemblages characterized by Globorotalia praescitula (from which it descended), G. zealandica, Globoturborotalita woodi, and Globorotaloides spp. at a level equivalent to MPC 5C (basal part) and correlative with its FO at Sites 516 (Berggren et al., 1983). Because of its setting in lower latitudes and under the influence of southward flowing subequatorial western boundary currents, waters over the Rio Grande Rise contained mixed subtropical and temperate elements in the late early to early middle Miocene, and it was possible to recognize several zones (M4-M7) based on the association of (prae)orbulinids and $G$. peripheroronda above the $G$. miozea (M4) Zone, which was restricted to a part of MPC 5C.

The G. miozea (NK4) Zone on the Kerguelen Plateau extends from MPC 5C (basal part) to a level (approximately) equivalent to the base of MPC 5AD (Site 747), the only site at which Neogloboquadrina nympha, the nominative form of the next higher zone, is thought to have a true initial occurrence; its appearance at Sites 748 and 751 is interpreted to occur at (above) a disconformity. Thus, Zone NK4 (Kerguelen Plateau) corresponds approximately to Zones M4-M7 (in part) of the South Atlantic (Berggren et al., 1983).

Neogloboquadrina nympha (NK5) Total Range Zone

Definition. Total range of nominate taxon.

Remarks. Neogloboquadrina nympha appeared in early middle Miocene faunas at Site 747 in association with Globorotalia miozea, G. praescitula, Globigerina bulloides, Globoturborotalita woodi, and Neogloboquadrina continuosa, from which it probably evolved. It was a common and distinct form in middle and early late Miocene faunas. Its LO is interpreted to occur at a level equivalent to MPC 5 at Sites 747 and 751 , whereas at Site 748 it appears to extend to a level questionably correlative with MPC 4A. Difficulties in interpreting the paleomagnetic record as well as with consistent identification of this taxon in the younger part of its range (when individuals typically exhibited a reduction in size) render the determination of the total extent of this zone uncertain at this time.

\section{Globorotalia scitula (NK6) Partial Range Zone}

Definition. Partial range of the nominate taxon between the $\mathrm{LO}$ of N. nympha (base) and FO of Neogloboquadrina pachyderma (top).

Remarks. Upper Miocene faunas of the Kerguelen Plateau are characterized by low diversity (5-7 species), erratic distribution (owing to the overwhelming abundance of biosiliceous forms and repeated migrations of the Polar Front across the region), and dominance of globigerinids ( $G$. bulloides) and globorotaliids $(G$. scitula). Globorotalia scitula is chosen as the nominate form of this zone because of its fairly common occurrence in the lower upper Miocene part of the record at 
Table 1. Location data for Leg 120 sites from which Neogene sediments were recovered.

\begin{tabular}{|c|c|c|c|l|}
\hline Site & Latitude & Longitude & Depth (mbsf) & \multicolumn{1}{|c|}{ Records on recovery } \\
\hline 747 & $54^{\circ} 48.68^{\prime} \mathrm{S}$ & $76^{\circ} 47.64^{\prime} \mathrm{E}$ & 1695 & $\begin{array}{l}\text { Essentially complete Neogene } \\
\text { section; several brief hiatuses }\end{array}$ \\
\hline 748 & $58^{\circ} 26.45^{\prime} \mathrm{S}$ & $7^{\circ} 58.89^{\prime} \mathrm{E}$ & 1290 & $\begin{array}{l}\text { Relatively complete Neogene } \\
\text { section; brief (2 m.y.) late } \\
\text { Miocene/early Pliocene hiatus and } \\
\text { somewhat longer (5 m.y.) late } \\
\text { early/middle Miocene hiatus. }\end{array}$ \\
\hline 751 & $57^{\circ} 43.56^{\prime} \mathrm{S}$ & $79^{\circ} 48.89^{\prime} \mathrm{E}$ & 1633.8 & $\begin{array}{l}\text { Relatively complete Neogene } \\
\text { section down to C5E }(\sim 18-19 \mathrm{Ma}) . \\
\text { Mid-Miocene ( 1-2 m.y.) and latest } \\
\text { Miocene/earliest Pliocene ( 0.5 } \\
\text { m.y.) hiatuses recorded. }\end{array}$ \\
\hline
\end{tabular}

Kerguelen. This zone corresponds roughly to the interval between MPC 5 (mid to upper part) and MPC 4A.

\section{Neogloboquadrina pachyderma (NK7) Total Range Zone}

Definition. Range of the nominate taxon from its initial occurrence (base) to the present.

Remarks. The initial appearance of Neogloboquadrina pachyderma is most clearly documented at Sites 747 and 751 where it appears within a predominantly biosiliceous ooze unit at a level equivalent to the middle part of MPC $4(\sim 7 \mathrm{Ma})$ and MPC $4 \mathrm{~A}(>8$ $\mathrm{Ma})$, respectively. At Site 748 , it appears (12 mbsf) in association with an unconformity separating lower Pliocene $(>4 \mathrm{Ma})$ and upper Miocene ( $<8 \mathrm{Ma}$; see Harwood and Maruyama, this volume; Lazarus et al., this volume). Typical $N$. pachyderma are not found in upper Miocene assemblages of the $N$. nympha or G. scitula zones; therefore, this zone is treated as a total, rather than as a partial, range zone.

\section{SITE 747}

Site 747 (proposed Site SKP-1) is located in the central part of the Kerguelen Plateau $\left(54^{\circ} 48.68^{\prime} \mathrm{S}, 76^{\circ} 47.64^{\prime} \mathrm{E}\right)$, about 500 $\mathrm{km}$ south of the present-day Polar Front (Antarctic Convergence) at $1695-\mathrm{m}$ water depth. Three holes were drilled at this site. A $295-\mathrm{m}$ section of Upper Cretaceous (Santonian) through Pleistocene (predominantly) pelagic carbonate ooze was recovered above $54 \mathrm{~m}$ of basalts. The sequence is punctuated by an approximately 8 -m-thick (182-190 mbsf) interval of lower Danian volcaniclastic breccias and cobbles, interpreted here as having been deposited as a massive debris flow.

The remaining (post-Danian) Cenozoic stratigraphic record is about $180 \mathrm{~m}$ thick and is essentially complete down to the upper lower Oligocene (equivalent to Subchron MPC 11N probably), where a disconformity at $\sim 170$ mbsf separates upper lower Oligocene from basal Oligocene (the hiatus is estimated to extend approximately from MPC $13 \mathrm{~N}$ to a level equivalent to Subchron MPC 11N, $\sim 32-35 \mathrm{Ma})$. A second disconformity at $\sim 173$ mbsf separates upper Eocene and lower Eocene sediments, and at $174.5 \mathrm{mbsf}$ a third disconformity separates the basal Eocene/uppermost Paleocene from lower Paleocene/upper Danian sediments. The stratigraphic interval above the Danian chalks is extremely condensed (the lower Eocene is essentially complete and represented by a stratigraphic thickness of $\sim 1.5 \mathrm{~m}$ between the unconformities at $\sim 173$ and 174.5 mbsf; see Berggren, chapter on Paleogene planktonic foraminifers, this volume).

\section{Hole 747A}

Hole $747 \mathrm{~A}$ was cored with the advanced hydraulic piston corer (APC) system to the upper Oligocene (151.5 mbsf), and the extended core barrel (XCB) system was then used to penetrate to a depth of $256 \mathrm{mbsf}$ (upper Campanian chalks).

\section{Hole 747B}

Six cores were drilled with the APC system at Hole 747B to a depth of $50.3 \mathrm{~m}$ (upper Miocene). A slight offset of about 2 $\mathrm{m}$ (shallower than Hole 47A) was noted and may have been caused by excessive heave of the ship during spudding in at Hole 747A.

\section{Hole 747C}

Hole $747 \mathrm{C}$ was rotary cored with the dual purpose of obtaining a more representative section of the condensed Oligocene to Paleocene sequence below $160 \mathrm{mbsf}$ (in Hole $747 \mathrm{~A}$ ) and of penetrating deeper in the Cretaceous sediments (below 256 mbsf in Hole 747A) in an attempt to reach basaltic basement. Both objectives were achieved.

\section{Neogene}

The Neogene is essentially complete at Site 747 , although several short hiatuses were delineated based on the integrated magnetobiostratigraphy. It is about $128 \mathrm{~m}$ thick (Fig. 2).

Pliocene-Pleistocene faunas are dominated by Neogloboquadrina pachyderma with lower numbers of Globigerina bulloides and G. falconensis. (Forms assigned to G. falconensis here may, in fact, represent kümmerform $G$. bulloides.) A notable occurrence of Globorotalia puncticulata was recorded in the course of preliminary studies based on corecatcher (CC) samples (Shipboard Scientific Party, 1989, pp. $107-109$ ), and is confirmed here based on additional studies. Rare individuals referred here to Globorotalia sphericomiozea were encountered in the interval from Section 120-747A$2 \mathrm{H}-\mathrm{CC}$ to $-3 \mathrm{H}-\mathrm{CC}$ (18.3-26.7 mbsf) in the Pliocene. At about $34 \mathrm{mbsf}$, an unconformity separates the lower Pliocene from the upper Miocene. Late Miocene faunas are characterized by Globorotalia scitula, Globigerina bulloides, Neogloboquadrina continuosa, N. pachyderma, and Globoturborotalita woodi.

Middle Miocene faunas are characterized by globigerinids ( $G$. bulloides), globoturborotalitids ( $G$. woodi), neogloboquadrinids ( $N$. nympha, $N$. continuosa), and globorotaliids (G. praescitula, G. miozea). The sequential appearance of $G$. miozea ( $\sim 83 \mathrm{mbsf})$ and $N$. nympha ( $\sim 72 \mathrm{mbsf})$ is used here for biostratigraphic subdivision of the middle Miocene and has been found applicable at Sites 748 and 751 as well, although ranges are punctuated by middle Miocene disconformities at those two sites. A notable occurrence of Globorotalia panda 
Table 2. Depths of Neogene magnetic polarity chronozones identified at Sites 747, 748, and 751 (Kerguelen Plateau).

\begin{tabular}{|c|c|c|c|c|c|c|}
\hline \multirow[b]{2}{*}{$\begin{array}{c}\text { Magnetic } \\
\text { polarity } \\
\text { chronozone }\end{array}$} & \multirow[b]{2}{*}{ Top } & \multirow[b]{2}{*}{ Base } & \multicolumn{4}{|c|}{ Depth (mbsf) } \\
\hline & & & $\begin{array}{l}\text { Hole } \\
747 \mathrm{~A}\end{array}$ & $\begin{array}{l}\text { Hole } \\
\text { 747B }\end{array}$ & $\begin{array}{c}\text { Hole } \\
748\end{array}$ & $\begin{array}{c}\text { Hole } \\
751\end{array}$ \\
\hline $2 \mathrm{~A}$ & $\mathrm{x}$ & $x$ & $\begin{array}{l}21.5 \\
26.0\end{array}$ & $\begin{array}{l}19.6 \\
23.4\end{array}$ & $\begin{array}{l}3.25 \\
6.95\end{array}$ & \\
\hline 3 & $x$ & $\mathrm{x}$ & & $\begin{array}{l}26.8 \\
32.7\end{array}$ & & $36.7^{(a)}$ \\
\hline $3 \mathrm{~A}$ & $x$ & $\mathrm{x}$ & & & $\begin{array}{l}13.55 \\
15.95\end{array}$ & 40.1 \\
\hline 4 & $x$ & $x$ & & & & \\
\hline $4 \mathrm{AN} 1$ & $x$ & $x$ & & & 25.05 & $\begin{array}{l}44.0 \\
48.4\end{array}$ \\
\hline 4AN2 & $x$ & $x$ & \begin{tabular}{|l|}
42.3 \\
43.05
\end{tabular} & $\begin{array}{l}36.20 \\
37.70\end{array}$ & $\begin{array}{l}13.55 \\
14.75\end{array}$ & $\begin{array}{l}42.70 \\
44.85\end{array}$ \\
\hline $4 \mathrm{AN} 3$ & $\mathrm{x}$ & $x$ & \begin{tabular}{|l|}
45.5 \\
46.6
\end{tabular} & $\begin{array}{l}38.20 \\
39.1\end{array}$ & $\begin{array}{l}14.95 \\
15.95\end{array}$ & $\begin{array}{l}45.00 \\
48.45\end{array}$ \\
\hline 5 & $\mathrm{x}$ & $\mathrm{x}$ & $\begin{array}{l}52.5- \\
55.0\end{array}$ & 50.0 & 17.25 & 51.7 \\
\hline $5 \mathrm{~A}$ & $x$ & $x$ & $\begin{array}{l}61.45 \\
65.4\end{array}$ & & & 99.9 \\
\hline $5 \mathrm{AA}$ & $x$ & $x$ & (b) & & & $\begin{array}{l}101^{(\mathrm{c})} \\
105.5\end{array}$ \\
\hline $5 \mathrm{AB}$ & $\mathrm{x}$ & $x$ & $\begin{array}{l}67.05 \\
68.90\end{array}$ & & & $\begin{array}{l}105.65 \\
108.10\end{array}$ \\
\hline $5 \mathrm{AC}$ & $x$ & $\mathrm{x}$ & $\begin{array}{l}70.10 \\
72.10\end{array}$ & & & \\
\hline $5 \mathrm{AD}$ & $\mathrm{x}$ & $x$ & $\begin{array}{l}72.75 \\
76.50\end{array}$ & & & \\
\hline $5 \mathrm{~B}$ & $x$ & $x$ & $\begin{array}{l}80.75 \\
82.10\end{array}$ & & & $\begin{array}{l}114.10 \\
116.70\end{array}$ \\
\hline $5 \mathrm{C}$ & $\mathrm{x}$ & $x$ & 91.05 & & & 123.30 \\
\hline 5D & $x$ & $x$ & 93.5 & & $\begin{array}{l}39.7 \\
42.0\end{array}$ & 132.8 \\
\hline $5 \mathrm{E}$ & $x$ & $x$ & $\begin{array}{l}100.0 \\
106.4\end{array}$ & & & 152.1 \\
\hline 6 & $x$ & $x$ & $\begin{array}{l}107.4 \\
112.9\end{array}$ & & 53.6 & \\
\hline $6 \mathrm{~A}$ & $x$ & $x$ & \begin{tabular}{|l|}
115.4 \\
119.1
\end{tabular} & & $\begin{array}{l}54.8 \\
57.5\end{array}$ & \\
\hline $6 \mathrm{AA}$ & $x$ & $x$ & $\begin{array}{l}120.6 \\
121.65\end{array}$ & & $58.8^{(d)}$ & \\
\hline $6 \mathrm{~B}$ & $\mathrm{x}$ & $x$ & \begin{tabular}{|l|}
123.1 \\
125.05
\end{tabular} & & $63.1^{\text {(d) }}$ & \\
\hline $6 C$ & $x$ & $x$ & $\begin{array}{l}125.7 \\
129.65\end{array}$ & & $\begin{array}{l}63.7 \\
68.2\end{array}$ & \\
\hline
\end{tabular}

Notes: Depths (in $\mathrm{m}$ below seafloor [mbsf]) are given only for intervals of unambiguously recovered polarity reversals. Data from Heider et al. (this volume) and Inokuchi and Heider (this volume). aprobably 3N2 (Thvera Subchron).

b Subchron C5AA is missing because of a hiatus in Hole 747A (Harwood and Maruyama, this volume).

${ }^{\mathrm{c}} \mathrm{An}$ interpretation of these events is uncertain because of inadequate biostratigraphic data (see Heider et al., this volume).

${ }^{\mathrm{d}}$ Subchrons C6AA and C6B are concatenated in Hole 748B (Inokuchi and Heider, this volume). 
Table 3. Magnetobiostratigraphic calibration of some Neogene planktonic foraminifer events at Site 747 (Kerguelen Plateau).

\begin{tabular}{|c|c|c|c|c|}
\hline Biostratigraphic event & $\begin{array}{l}\text { Core, section, } \\
\text { interval }(\mathrm{cm})\end{array}$ & $\begin{array}{l}\text { Depth } \\
\text { (mbsf) }\end{array}$ & $\begin{array}{l}\text { Paleomagnetic } \\
\text { calibration }\end{array}$ & Remarks \\
\hline $\begin{array}{l}\text { 1. FO Neogloboquadrina } \\
\text { pachyderma }\end{array}$ & $\begin{array}{l}4 \mathrm{H}-6,20-24 \mathrm{~cm}, \text { to } \\
4 \mathrm{H}-\mathrm{CC}\end{array}$ & $35.9-37.5$ & C4N2 & $\begin{array}{l}\text { Just below } \\
\text { unconformity }\end{array}$ \\
\hline $\begin{array}{l}\text { 2. LO Globorotalia } \\
\text { praescitula }\end{array}$ & $\begin{array}{l}7 \mathrm{H}-4,40-44 \mathrm{~cm} \text { to } \\
7 \mathrm{H}-6,40-44 \mathrm{~cm}\end{array}$ & $61.4-64.4$ & C5A & \\
\hline $\begin{array}{l}\text { 3. FO Neogloboquadrina } \\
\text { nympha }\end{array}$ & $\begin{array}{l}8 \mathrm{H}-2,40-44 \mathrm{~cm} \text { to } \\
8 \mathrm{H}-3,40-44 \mathrm{~cm}\end{array}$ & $67.9-69.4$ & $\begin{array}{l}\mathrm{C} 5 \mathrm{AB} \\
\text { (base) }\end{array}$ & $\begin{array}{l}\text { Reinterpretation of } \\
\text { mid-Miocene } \\
\text { magnetostrati- } \\
\text { graphy (Harwood } \\
\text { and Maruyama, } \\
\text { this volume) }\end{array}$ \\
\hline $\begin{array}{l}\text { 4. LO Globorotalia } \\
\text { zealandica }\end{array}$ & $\begin{array}{l}8 \mathrm{H}-\mathrm{CC} \\
\text { to } \\
9 \mathrm{H}-2,40-44 \mathrm{~cm}\end{array}$ & $75.5-77.4$ & $\mathrm{C} 5 \mathrm{AD}$ & $\begin{array}{l}\text { Reinterpretation of } \\
\text { mid-Miocene } \\
\text { magnetostrati- } \\
\text { graphy (Harwood } \\
\text { and Maruyama, } \\
\text { this volume) }\end{array}$ \\
\hline $\begin{array}{l}\text { 5. LO Paragloborotalia } \\
\text { incognita }\end{array}$ & $\begin{array}{l}9 \mathrm{H}-4,40-44 \mathrm{~cm} \\
\text { to } \\
9 \mathrm{H}-6,40-44 \mathrm{~cm}\end{array}$ & $79.9-82.9$ & C5B & \\
\hline $\begin{array}{l}\text { 6. FO Globorotalia } \\
\text { miozea }\end{array}$ & $\begin{array}{l}9 \mathrm{H}-6,40-44 \mathrm{~cm} \\
\text { to } \\
9 \mathrm{H}-8,40-44 \mathrm{~cm}\end{array}$ & $82.9-84.4$ & $\begin{array}{l}\text { Just above } \\
\text { top C5C } \\
\text { (85.3) }\end{array}$ & \\
\hline $\begin{array}{l}\text { 7. LO Catapsydrax } \\
\text { unicavus }\end{array}$ & $\begin{array}{l}10 \mathrm{H}-\mathrm{CC} \\
\text { to } \\
11 \mathrm{H}-1,40-44 \mathrm{~cm}\end{array}$ & $94.5-94.9$ & $\begin{array}{c}\text { C5D } \\
\text { (upper part) }\end{array}$ & \\
\hline $\begin{array}{l}\text { 8. LO Catapsydrax } \\
\text { dissimilis }\end{array}$ & $\begin{array}{l}11 \mathrm{H}-4,40-44 \mathrm{~cm} \text { to } \\
11 \mathrm{H}-6,40-44 \mathrm{~cm}\end{array}$ & $95.4-102.4$ & $\begin{array}{l}\text { Just above top C5E } \\
\text { (at 100) }\end{array}$ & \\
\hline
\end{tabular}

Table 4. Stratigraphic position and magnetobiostratigraphic calibration of main Neogene chronostratigraphic boundaries at Site 747.

\begin{tabular}{|c|c|c|c|}
\hline $\begin{array}{c}\text { Chronostratigraphic } \\
\text { boundary }\end{array}$ & Biostratigraphic event & Depth (mbsf) & $\begin{array}{l}\text { Paleomagnetic } \\
\text { calibration }\end{array}$ \\
\hline 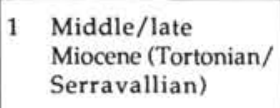 & $\begin{array}{l}\text { LO N. nympha in } \\
\text { (upper part of) C5N }\end{array}$ & $\sim 55$ & Base $\mathrm{C} 5 \mathrm{~N}$ \\
\hline $\begin{array}{l}\text { 2. Early/middle } \\
\text { Miocene (Langhian/ } \\
\text { Burdigalian) }\end{array}$ & $\begin{array}{l}\text { FO G. miozea } \\
\text { (1 m higher) }\end{array}$ & 85.35 & Top C5CN \\
\hline $\begin{array}{l}\text { 3. Oligocene/ } \\
\text { Miocene } \\
\text { (Aquitanian/ } \\
\text { Chattian) }\end{array}$ & $\begin{array}{l}\text { LO } \\
\text { G. euapertura } \\
\text { ( } 2 \text { m below })\end{array}$ & 127.9 & $\mathrm{C} 6 \mathrm{CN} 2$ \\
\hline
\end{tabular}

Note: Chronostratigraphic boundary calibrations to paleomagnetic stratigraphy based on Berggren et al. (1985).

(61-65 mbsf) in high numbers is noted over a short stratigraphic interval within the $N$. nympha Zone.

Early Miocene faunas were moderately diverse and dominated by catapsydracids ( $C$. unicavus, $C$. dissimilis), Globorotaloides suteri, globorotaliids ( $G$. zealandica, $G$. praescitula), paragloborotaliids ( $P$. incognita- $P$. semivera group), and Globoturborotalita woodi. The sequential appearance of $P$. incognita $(\sim 120 \mathrm{mbsf})$ and $G$. praescitula $(\sim 104 \mathrm{mbsf})$ is used here (as well as at Site 748) for biostratigraphic subdivision of the lower Miocene. Although difficult to distinguish from $G$. woodi in some instances, the trilobate form $G$. brazieri is characteristic for the lower part of the middle Miocene. Large, robust forms with thickened apertural rims characterize the upper Oligocene-lower Miocene of Hole 747A (as well as Hole 748B) and are referred here to Globigerina $\mathrm{cf}$. labiacrassata.

The Oligocene/Miocene boundary is drawn at $\sim 128 \mathrm{mbsf}$ in Hole $747 \mathrm{~A}$, based on the identification of MPC 6C (Heider, in 
Table 5. Magnetobiostratigraphic calibration of some Neogene planktonic foraminifer events in Hole 748B.

\begin{tabular}{|c|c|c|c|c|}
\hline Biostratigraphic event & $\begin{array}{l}\text { Core, section, } \\
\text { interval }(\mathrm{cm})\end{array}$ & $\begin{array}{l}\text { Depth } \\
\text { (mbsf) }\end{array}$ & $\begin{array}{l}\text { Paleomagnetic } \\
\text { calibration }\end{array}$ & Remarks \\
\hline $\begin{array}{l}\text { 1. LO Neogloboquadrina } \\
\text { nympha }\end{array}$ & $\begin{array}{l}3 \mathrm{H}-\mathrm{CC} \\
\text { to } \\
4 \mathrm{H}-1,40-44 \mathrm{~cm}\end{array}$ & $19.1-19.5$ & $\mathrm{C} 5 \mathrm{~N}$ & \\
\hline $\begin{array}{l}\text { 2. FO Globorotalia } \\
\text { zealandica }\end{array}$ & $\begin{array}{l}6 \mathrm{H}-\mathrm{CC} \text { and } \\
7 \mathrm{H}-1,40-44 \mathrm{~cm}\end{array}$ & $47.6-48.0$ & C5E & $\begin{array}{l}\text { Interpreted (on less } \\
\text { reliable data) as } \\
\text { being 5E }\end{array}$ \\
\hline $\begin{array}{l}\text { 3. FO Globorotalia } \\
\text { praescitula }\end{array}$ & $\begin{array}{l}6 \mathrm{H}-\mathrm{CC} \text { and } 7 \mathrm{H}-1, \\
40-44 \mathrm{~cm}\end{array}$ & $47.6-48.0$ & C5E & $\begin{array}{l}\text { Interpreted (on less } \\
\text { reliable data) as } \\
\text { being 5E }\end{array}$ \\
\hline $\begin{array}{l}\text { 4. LO Globigerina } \\
\text { euapertura }\end{array}$ & $\begin{array}{l}8 \mathrm{H}-2,40-44 \mathrm{~cm} \\
\text { to } \\
8 \mathrm{H}-6,40-44 \mathrm{~cm}\end{array}$ & 63.565 .0 & $\mathrm{C} 6 \mathrm{C}$ & \\
\hline
\end{tabular}

Table 6. Stratigraphic position and magnetobiostratigraphic calibration of Oligocene/Miocene boundary at Site 748 .

\begin{tabular}{|c|c|c|c|c|}
\hline $\begin{array}{c}\text { Chronostratigraphic } \\
\text { boundary }\end{array}$ & $\begin{array}{c}\text { Biostratigraphic } \\
\text { event }\end{array}$ & $\begin{array}{c}\text { Core, section, } \\
\text { interval }(\mathrm{cm})\end{array}$ & $\begin{array}{c}\text { Depth } \\
\text { (mbsf) }\end{array}$ & $\begin{array}{c}\text { Magnetostrati- } \\
\text { graphic calibration }\end{array}$ \\
\hline Oligocene/Miocene & $\begin{array}{c}\text { Closely associated } \\
\text { with LO } \\
\text { G. euapertura }\end{array}$ & $\begin{array}{c}8 \mathrm{H}-\mathrm{CC} \\
\text { to } \\
\begin{array}{c}\mathrm{H}-1,40-44 \\
\text { on }\end{array}\end{array}$ & $\begin{array}{c}66.6-67.0 \\
{[66.8]}\end{array}$ & Midpoint C6CN2 \\
& & & \\
\hline
\end{tabular}

Note: The depth given in brackets is the midpoint between two adjacent sample depths.

Shipboard Scientific Party, 1989; see also Heider et al., this volume) and correlation with the Global Polarity Time Scale (GPTS) of Berggren et al. (1985). The LOs of Globigerina euapertura and Tenuitella munda have been observed in close association with this level.

\section{SITE 748}

Site 748 (proposed Site SKP-3C) is a reentry site located on the Southern Kerguelen Plateau in the western part of the Raggatt Basin, east of Banzare Bank ( $\left.58^{\circ} 26.45^{\prime} \mathrm{S}, 78^{\circ} 58.89^{\prime} \mathrm{E}\right)$ at a water depth of about $1290 \mathrm{~m}$. Three holes were drilled at this site. An 899-m-thick sequence of upper Albian to Turonian through Pleistocene sediments overlay several meters of highly altered basalt flows, which overlay about $30 \mathrm{~m}$ of fragments of variegated clays; brown smectitic, sideritic clays; and highly altered basalt fragments of indeterminate origin and age.

The Cenozoic stratigraphic record of Site 748 is represented by about $410 \mathrm{~m}$ and spans the lower upper Paleocene (equivalent to Zones P3 or P4, about $61 \mathrm{Ma}$ ) to the Holocene. The Paleocene lies unconformably upon the upper Maestrichtian (Nephrolithus frequens Zone; $67-68 \mathrm{Ma}$ ), suggesting a hiatus of about 6-7-m.y. duration. At least one unconformity is detectable by planktonic foraminifer biostratigraphy and is situated at about 38 mbsf (between Cores 120-748B-5H and $-6 \mathrm{H})$. This unconformity separates middle Miocene from upper lower Miocene sediments. The hiatus is estimated to span about $5 \mathrm{~m}$.y. (from 12-17 Ma). The presence of other hiatuses of short duration at Site 748 is discussed elsewhere (Shipboard Scientific Party, 1989, p. 205, and p. 193, table 6; Lazarus et al., this volume).

\section{Hole 748A}

Nineteen meters of sediment were recovered from Hole $748 \mathrm{~A}$, which ended in lower Pliocene diatomaceous oozes containing ice-rafted detritus.

\section{Hole 748B}

The APC was used in Hole 748B to a depth of $180.6 \mathrm{mbsf}$ (Core 120-748B-18H) where middle Eocene (Acarinina collactea Zone of Stott and Kennett, 1990, $\cong$ Zone P12) cherts were encountered. Core recovery within this interval was essentially $100 \%$. Coring with the XCB then continued through alternate nannofossil oozes and chert stringers (resulting in recoveries ranging from $0 \%$ to $45 \%$ ) and ending in the same $A$. collactea Zone as the APC coring at 225.1 mbsf (Core 120748B-25X).

\section{Hole 748C}

Rotary coring (RCB) began at 173 mbsf (overlapping with Core $120-748 \mathrm{~B}-20 \mathrm{H}$ ) and continued to a terminal depth of 935 mbsf (Core 120-748B-87R) in weathered and altered Lower Cretaceous basalt flows. Cores $120-748 B-1 R$ to $-25 R$ represent the Cenozoic; lower upper Paleocene nannofossil oozes, calcareous grainstones, and cherts lie unconformably on upper Maestrichtian glauconitic, bioclastic packstones in the interval between Cores 120-748B-26R-CC and 120-748B-27R$\mathrm{CC}$ at about 410 mbsf.

\section{Neogene}

The Neogene (Miocene, Pliocene, Pleistocene, and Holocene) is represented by about $67 \mathrm{~m}$ of sediments (Fig. 3). 
Table 7. Paleomagnetic calibration of some Neogene planktonic foraminifer events at Site 751.

\begin{tabular}{|c|c|c|c|c|}
\hline Biostratigraphic event & $\begin{array}{l}\text { Core, section, } \\
\text { interval }(\mathrm{cm})\end{array}$ & $\begin{array}{l}\text { Depth } \\
\text { (mbsf) }\end{array}$ & $\begin{array}{l}\text { Paleomagnetic } \\
\text { calibration }\end{array}$ & Remarks \\
\hline $\begin{array}{l}\text { 1. FO Neogloboquadrina } \\
\text { pachyderma }\end{array}$ & $\begin{array}{l}6 \mathrm{H}-2,18-22 \mathrm{~cm} \text { to } \\
6 \mathrm{H}-3,18-22 \mathrm{~cm}\end{array}$ & $\begin{array}{c}44.5 \text { to } \\
46.0\end{array}$ & $\mathrm{C} 4 \mathrm{AN}$ & $\begin{array}{l}\text { just below } \\
\text { disconformity }\end{array}$ \\
\hline $\begin{array}{l}\text { 2. LO Neogloboquadrina } \\
\text { nympha }\end{array}$ & $\begin{array}{l}6 \mathrm{H}, \mathrm{CC} \text { to } \\
7 \mathrm{H}-1,130-134 \mathrm{~cm}\end{array}$ & $\begin{array}{l}52.2 \text { to } \\
53.5\end{array}$ & $\mathrm{C} 5 \mathrm{~N}$ & \\
\hline $\begin{array}{l}\text { 3. LO Globorotalia } \\
\text { zealandica }\end{array}$ & $\begin{array}{l}14 \mathrm{H}-4,32-36 \mathrm{~cm} \text { to } \\
14 \mathrm{H}-3,32-36 \mathrm{~cm}\end{array}$ & $\begin{array}{l}123.5 \text { to } \\
122.0\end{array}$ & $\begin{array}{l}\mathrm{C5C} \\
\text { (base) }\end{array}$ & \\
\hline $\begin{array}{l}\text { 4. FO Globorotalia } \\
\text { miozea }\end{array}$ & $\begin{array}{l}14 \mathrm{H}-3,32-36 \mathrm{~cm} \text { to } \\
14 \mathrm{H}-04,32-36 \mathrm{~cm}\end{array}$ & $\begin{array}{l}122.0 \text { to } \\
123.5\end{array}$ & $\begin{array}{c}\mathrm{C} 5 \mathrm{C} \\
\text { (base) }\end{array}$ & \\
\hline $\begin{array}{l}\text { 5. LO Globorotaloides } \\
\text { suteri }\end{array}$ & $\begin{array}{l}15 \mathrm{H}-5,32-36 \mathrm{~cm} \text { to } \\
15 \mathrm{H}-4,32-36 \mathrm{~cm}\end{array}$ & $\begin{array}{l}133.0 \text { to } \\
134.5\end{array}$ & $\begin{array}{c}\text { C5D } \\
\text { (upper part) }\end{array}$ & \\
\hline $\begin{array}{l}\text { 6. FO Globorotalia } \\
\text { zealandica }\end{array}$ & $\begin{array}{l}16 \mathrm{H}-\mathrm{CC} \text { to } \\
17 \mathrm{H}-01,40-44 \mathrm{~cm}\end{array}$ & $\begin{array}{l}147.2 \text { to } \\
147.5\end{array}$ & & $\begin{array}{l}\text { No paleomagnetic } \\
\text { data; between } 5 \mathrm{D} \\
\text { and } 5 \mathrm{E}\end{array}$ \\
\hline $\begin{array}{l}\text { 7. LO Paragloborotalia } \\
\text { incognita }\end{array}$ & $\begin{array}{l}17 \mathrm{H}-1,32-36 \mathrm{~cm} \text { to } \\
16 \mathrm{H}-\mathrm{CC}\end{array}$ & $\begin{array}{l}147.5 \text { to } \\
147.2\end{array}$ & & $\begin{array}{l}\text { No paleomagnetic } \\
\text { data; between } 5 \mathrm{D} \\
\text { and } 5 \mathrm{E}\end{array}$ \\
\hline $\begin{array}{l}\text { 8. FO Globorotalia } \\
\text { praescitula }\end{array}$ & $\begin{array}{l}17 \mathrm{H}-6,32-36 \mathrm{~cm} \text { to } \\
17 \mathrm{H}-7,32-36 \mathrm{~cm}\end{array}$ & $\begin{array}{l}155.0 \text { to } \\
156.5\end{array}$ & $\begin{array}{c}\text { C5E } \\
\text { (mid to upper } \\
\text { half) }\end{array}$ & \\
\hline
\end{tabular}

Table 8. Stratigraphic level and paleomagnetic calibration of early/ middle Miocene boundary, Hole 751 .

\begin{tabular}{|c|c|c|c|}
\hline $\begin{array}{c}\text { Chronostratigraphic } \\
\text { boundary }\end{array}$ & $\begin{array}{c}\text { Biostratigraphic } \\
\text { event }\end{array}$ & $\begin{array}{c}\text { Depth } \\
\text { (mbsf) }\end{array}$ & $\begin{array}{c}\text { Paleomagnetic } \\
\text { calibration }\end{array}$ \\
\hline Early/middle Miocene & $\begin{array}{l}\text { Closely associated } \\
\text { with FO G. miozea }\end{array}$ & 113 & Top C5C \\
\hline
\end{tabular}

The Oligocene/Miocene boundary is associated here with the middle of three characteristic normal polarity subchrons (at about 67 mbsf) (Shipboard Scientific Party, 1989, p. 201) identified as Subchron C6CN.

The Pliocene and Pleistocene faunas (approximately 12-0 mbsf) are dominated (almost exclusively) by Neogloboquadrina pachyderma; sporadic and lower abundances of Globigerina bulloides and Globorotalia scitula punctuate this interval.

Middle and upper Miocene faunas are characterized by fairly high abundances of Globigerina bulloides and the persistent association of Globorotalia scitula, Neogloboquadrina continuosa, and Globigerina uvula. Neogloboquadrina pachyderma appears in association with an unconformity at 12 mbsf that separates the lower Pliocene $(>4 \mathrm{Ma})$ from upper Miocene ( $\sim 8 \mathrm{Ma})$ sediments. Neogloboquadrina nympha, a middle to early late Miocene isomorph of the characteristically low-latitude $N$. acostaensis, ranges from about 38.1 to 19.3 mbsf (Cores 120-748B-5H-CC to $-4 \mathrm{H}-1,40-44 \mathrm{~cm}$ ).

An unconformity at about $38 \mathrm{~m}$ separates middle Miocene and upper lower Miocene nannofossil oozes; the duration of the hiatus is estimated at about $5 \mathrm{~m} . \mathrm{y}$., from 17 to $12 \mathrm{Ma}$ (see Shipboard Scientific Party, 1989, p. 180, and discussion below). The lower Miocene sediments (Cores 120-748B-6H-1, $40-44 \mathrm{~cm}$, to $-8 \mathrm{H}-\mathrm{CC}$; around $38.5-67 \mathrm{mbsf}$ ) are characterized by globigerinid ( $G$. woodi), catapsydracid ( $C$. unicavusdissimilis group and Globorotaloides suteri), and paragloboro- taliid ( $P$. incognita) and Globorotalia (G. zealandica, $G$. praescitula) faunas.

Lower Miocene faunas are also characterized by the trilobate Globigerina brazieri (to Sample 120-748B-7H-4, 40-44 $\mathrm{cm} ; \sim 52.5 \mathrm{mbsf}$ ) and the large, generally evolute, and thickly rimmed G. cf. labiacrassata (to Sample 120-748B-8H-2, $40-44 \mathrm{~cm} ; 59 \mathrm{mbsf})$. As at Site 747 , the LO of Globigerina euapertura (Sample 120-748B-8H-6, 40-44 cm; $65 \mathrm{mbsf}$ ) is closely associated with MPC $6 \mathrm{C}$.

\section{SITE 751}

Site 751 (proposed Site SKP-2C) is situated in the central part of the Raggatt Basin (southern part of the Kerguelen Plateau; $57^{\circ} 43.56^{\prime} \mathrm{S}, 79^{\circ} 48.89^{\prime} \mathrm{E}$ ) at a water depth of $1634 \mathrm{~m}$. The site lies some $900 \mathrm{~km}$ south of the present-day Polar Front and was drilled to obtain a high-resolution Neogene and Paleogene stratigraphic section above the carbonate compensation depth (CCD).

About $166 \mathrm{~m}$ of alternating and mixed calcareous and biogenic siliceous (predominantly diatomaceous) oozes were recovered in an expanded section that extends, interrupted by several disconformities, from the lower Miocene to the Pleistocene. Because of time constraints, the remainder of the Miocene and underlying Paleogene sediments were not recovered.

\section{Neogene}

The most striking feature of the planktonic foraminifer faunal record obtained in Hole 751A is the low diversity, which ranges from about 9-10 species in the lower Miocene to a single form, Neogloboquadrina pachyderma, in much of the Pliocene and Pleistocene (Fig. 4).

The oldest faunas in Hole 751A were dominated by globigerinids ( $G$. cf. labiacrassata), globoturborotalitids $(G$. brazieri), and catapsydracids $(C$. dissimilis) as well as Globorotaloides suteri. The sequential appearance of Paragloborota- 


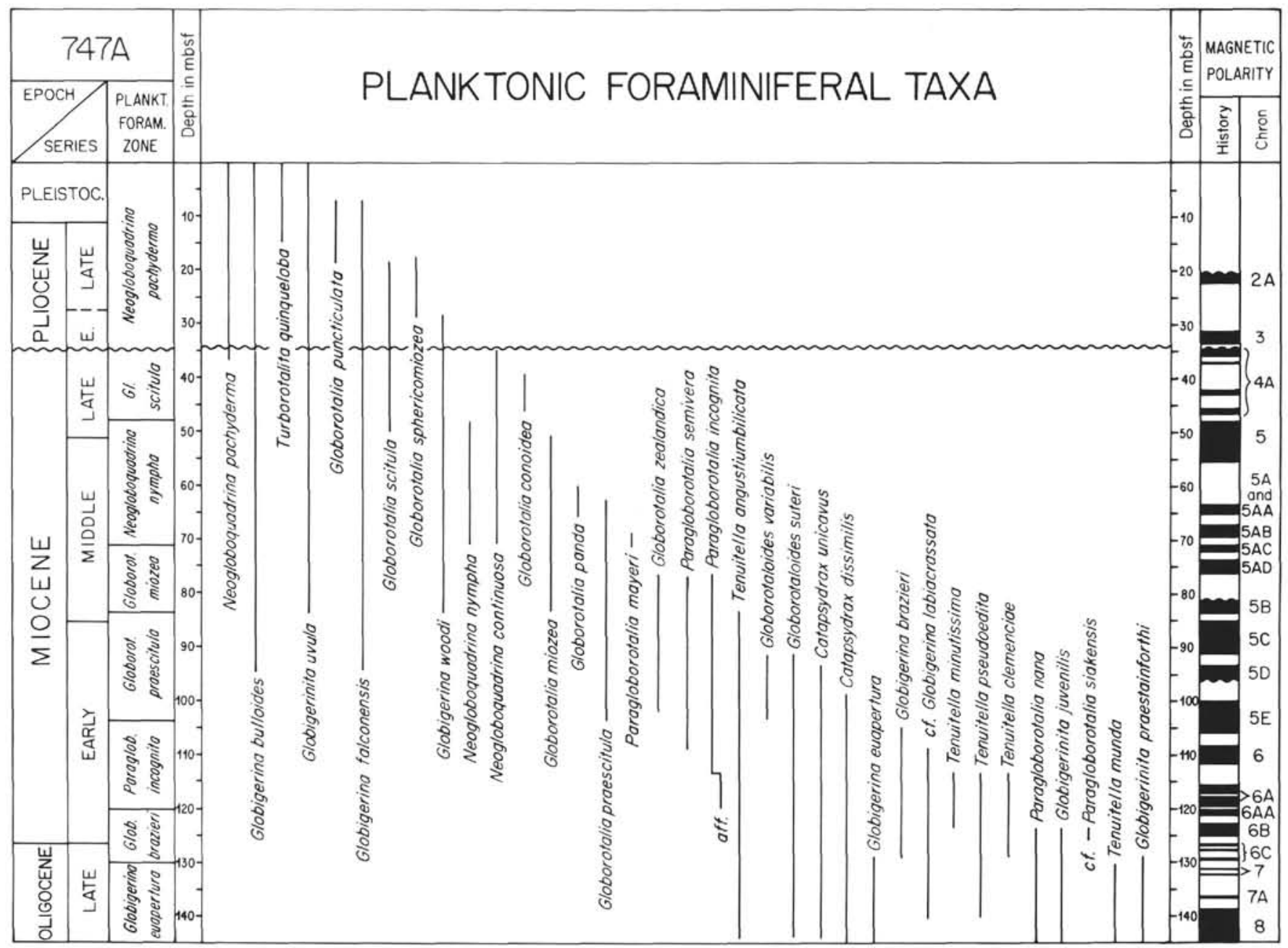

Figure 2. Stratigraphic range of Neogene planktonic foraminifer taxa, Hole 747A.

lia incognita and $P$. semivera ( $\sim 160 \mathrm{mbsf})$, Globorotalia praescitula ( $\sim 155 \mathrm{mbsf})$, and G. zealandica ( $\sim 147 \mathrm{mbsf})$ mirrors that observed at Sites 747 and 748 and aids biostratigraphic subdivision of this part of the lower Miocene.

Globorotalia miozea appears at $122 \mathrm{mbsf}$, about $1.5 \mathrm{~m}$ above the disappearance of $G$. zealandica. Together with $G$. praescitula and $G$. woodi, it dominated late early and early middle Miocene assemblages. A middle Miocene disconformity at 109-110 mbsf (between Cores 120-751A-12 $\mathrm{H}$ and $13 \mathrm{H}$ ) abruptly ends the $G$. praescitula $-G$. miozea faunas. These are replaced by a low diversity (3-4 species generally) assemblage dominated by Neogloboquadrina nympha and (in younger parts of the middle-late Miocene) by $G$. woodi.

Neogloboquadrina nympha ranges at least as high as 53.5 mbsf, although its distribution between 95 and 54 mbsf is sporadic and it is present in only small amounts.

There is an approximately $10 \mathrm{~m}$ thick sequence of diatomaceous-calcareous oozes ( $\sim 42-52$ mbsf $)$, in which the abundance of planktonic foraminifers varies considerably, probably a reflection of fluctuations in the Polar Front over the site. This predominantly biosiliceous ooze is overlain disconformably by Pliocene-Pleistocene (predominantly) biosiliceous oozes in which Neogloboquadrina pachyderma is relatively common. Below the unconformity, the dominant form at those levels at which planktonic foraminifers are common to abundant is Globigerina bulloides. Neogloboquadrina pachyderma makes its initial appearance several meters below the unconformity in Sample 120-751A-6H-2, $18.22 \mathrm{~cm}(44.5$ mbsf), the age of which is estimated here at $>8 \mathrm{Ma}$, assuming that the interpretation of the normal polarity event between 44 and 48.5 mbsf as MPC $4 \mathrm{~A}$ is correct. It occurs together with rare forms referred here to $N$. continuosa, from which it probably evolved.

Above the unconformity at $\sim 41$ mbsf, Neogloboquadrina pachyderma is, essentially, the lone planktonic foraminifer at this site to occur over the past $5 \mathrm{~m}$.y. in what is essentially a biosiliceous (diatomaceous) ooze.

\section{MAGNETOBIOSTRATIGRAPHY}

It has been possible to calibrate several planktonic foraminifer bioevents (and zonal boundaries) to the paleomagnetic stratigraphy. A brief summary of the main calibration points is presented below; these events are listed also in Tables 3, 5, and 7 accompanying the description of site biostratigraphies. The sub-bottom depths of Neogene magnetic polarity chronozones identified at Sites 747, 748, and 751 are listed in Table 2 above. Ages of the foraminifer FODs and LODs are obtained by interpolating between boundaries and using the GPTS of Berggren et al. (1985).

\section{Early Miocene}

The close association of the LO of Globigerina euapertura (= base Zone NK1) with MPC C6C at Sites 747 and 748 may serve as a means of correlation to the low latitudes where the 


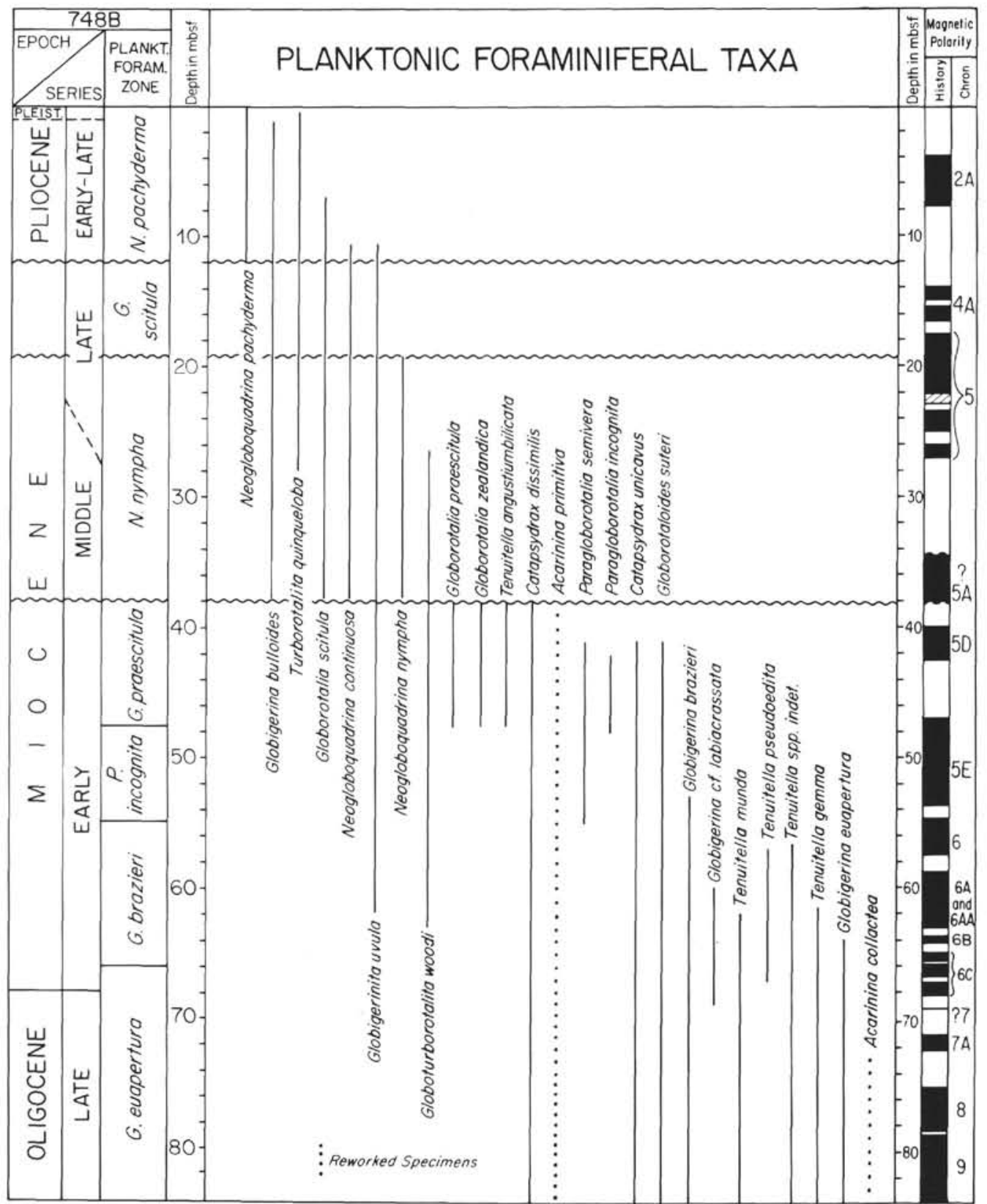

Figure 3. Stratigraphic range of Neogene planktonic foraminifer taxa, Hole 748B.

FO of Globorotalia kugleri has been found to correlate with MPC C6C and used to denote a level within Zone "N4" of Blow (1969) (see Berggren and Miller, 1988) and the base of Zone M1 (Berggren et al., 1983) and to identify the Oligocene/ Miocene boundary (Berggren et al., 1985).

The NK1/NK2 boundary (= FO $P$. incognita) is associated with MPC 6A at Sites 747 and 748, somewhat higher than its interpreted correlation with MPC 6B at Site 516 on the Rio Grande Rise (Berggren et al., 1983). This difference may be attributed to one or a combination of the following: (1) strong dissolution during the early Miocene at Kerguelen Plateau, resulting in poor preservation of, and concomitant difficulty in, clearly identifying the earliest forms of this taxon; and (2) difficulties in correctly identifying the magnetic polarity chronozones at Sites 516 and 747 .

The NK2/NK3 boundary (= FO Globorotalia praescitula) appears to correlate with MPC'5E at Sites 747, 748, and
751, somewhat earlier than its (interpreted) correlation with MPC 5D at Site 516 on the Rio Grande Rise. The FO of this taxon has recently been recognized in mid-MPC 5E $(\sim 18.8$ Ma) at northeast North Atlantic DSDP Site 608 (Miller et al., 1991). The $\mathrm{Sr}$ isotope record at Site 516 exhibits greater scatter than that of Site 608 for the lower Miocene (21-15 $\mathrm{Ma})$, and the problem has been ascribed to the problem in correctly interpreting the magnetic polarity stratigraphy at Site 516 (Miller et al., 1991). It would appear that the correlation of the FO of $G$. praescitula and MPC $5 \mathrm{E}$ is now rather well established.

The NK3/NK4 boundary (= FO Globorotalia miozea) corresponds to a level at the base of MPC 5C at Site 751, correlative with its FO at Site 516. The LO of $G$. zealandica occurs at the same level as the FO of $G$. miozea (base MPC 5C) at Site 751 , whereas at Site 747 it extends into the middle Miocene to Subchron C5AD (i.e., about 2 m.y. younger). 


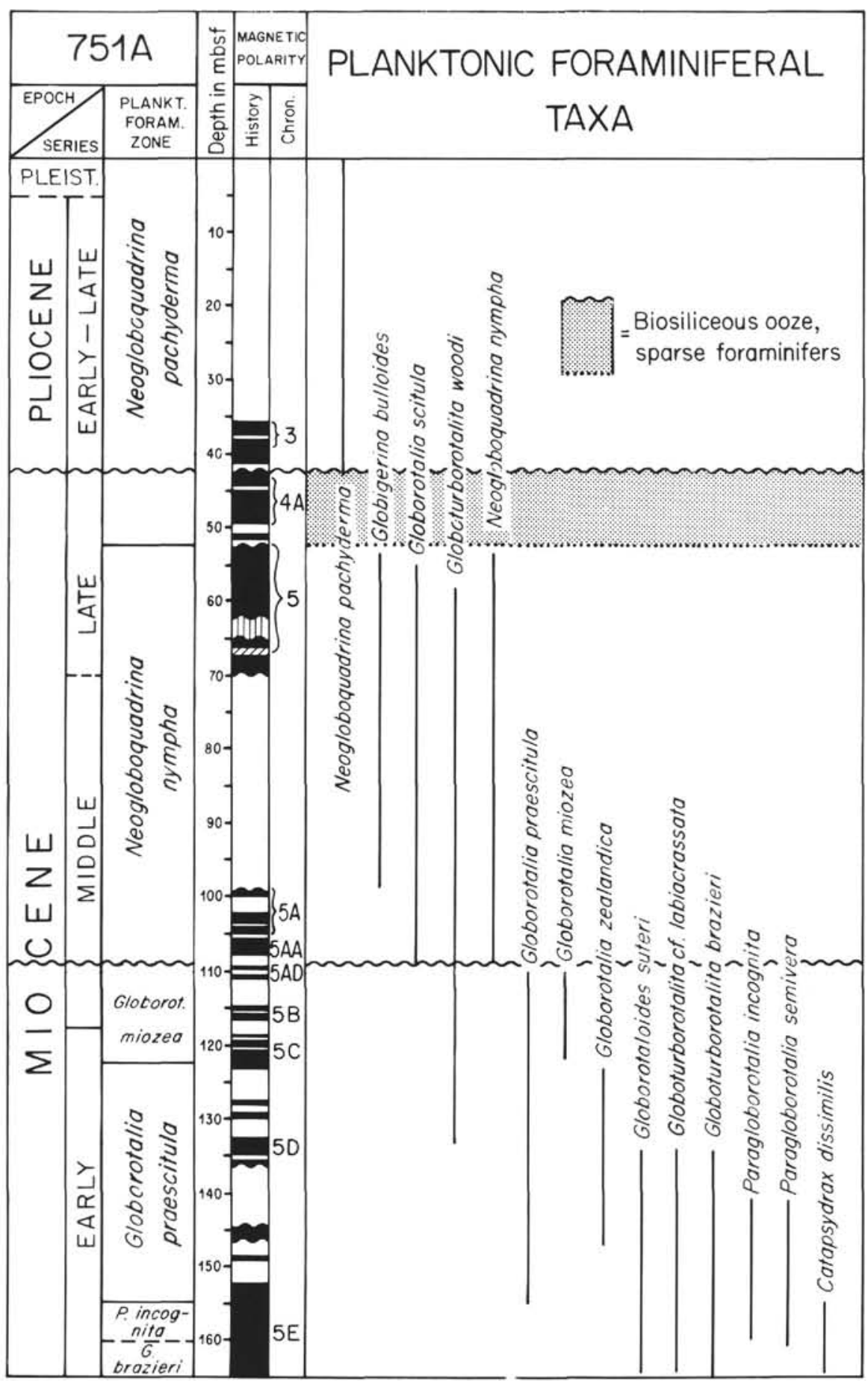

Figure 4. Stratigraphic range of Neogene planktonic foraminifer taxa, Hole 751A.

\section{Middle Miocene}

There are few distinct biostratigraphic events in the middle Miocene. The FO of Neogloboquadrina nympha is used here to denote the NK4/NK5 boundary. At Site 747 this event occurs near the base of MPC 5ABN. At Site 608 (northeast Atlantic), the FO of $N$. nympha is in a sample assigned to MPC 5AAR, just above C5AB (Miller et al., 1991); the apparent stratigraphic offset is within the sampling interval error. The $\mathrm{LO}$ of Globorotalia praescitula occurs in association with (concatenated) MPCs 5A-5AA at Site 747.
Although it does not provide a definitive calibration to the GPTS, it suggests an LO roughly equivalent to the younger part of the G. fohsi lineage of low latitudes, somewhat younger than that indicated by Kennett and Srinivasan (1983, pp. 8 and 104).

\section{Late Miocene}

The LO of Neogloboquadrina nympha is associated with MPC $5(\sim 9 \mathrm{Ma})$ at Sites 747,748 , and 751 . The FO of Neogloboquadrina pachyderma is interpreted to occur in association with MPC 4 at about $7 \mathrm{Ma}$ and MPC 4A at about 
$8+$ Ma at Sites 747 and 751 , respectively. I have not observed it at older levels (i.e., in MPC 5).

\section{CHRONOSTRATIGRAPHY}

A magnetobiostratigraphically calibrated framework for Neogene chronostratigraphy was developed by Berggren et al. (1985). Low-latitude calcareous plankton zonal biostratigraphies were correlated to the Neogene magnetostratigraphy and an estimated magnetobiochronology suggested.

Neogene planktonic foraminifer faunas of the Kerguelen Plateau exhibited few elements in common with those of low latitudes, and the process of identifying polarity patterns correctly at high latitudes is fraught with difficulties, which are discussed at greater length in a separate paper (Berggren et al., this volume). Despite these difficulties, we have been able to identify a number of biostratigraphic events that appear to be useful in high-latitude austral stratigraphy and which may serve to denote characteristic parts of the standard polarity record and then permit fairly precise correlation with standard low-latitude biostratigraphies and, ultimately, the standard chronostratigraphy of the Neogene.

The estimated depth of some Neogene chronostratigraphic boundaries on the Kerguelen Plateau is shown in Tables 4, 6, and 8.

\section{Oligocene/Miocene Boundary}

The Oligocene/Miocene boundary has been calibrated with Subchron C6CN2 (Berggren et al., 1985) and associated with the FO of Globorotalia kugleri and the LO of the calcareous nannoplankton Reticulofenestra bisecta. On Kerguelen Plateau, however, the LO of Globigerina euapertura has been found to be closely associated with Chron C6CN at Sites 747 and 748 , and it may serve as a useful criterion in recognizing the (approximate) position of the base of the Neogene in high austral latitudes.

\section{Early/Middle Miocene Boundary}

The early/middle Miocene boundary corresponds to the end of Subchron C5C ( 16.2 Ma) (Berggren et al., 1985). This event was recognized at Sites $747(\sim 85 \mathrm{mbsf})$ and $751(\sim 113$ mbsf) and is associated approximately with the FO of Globorotalia miozea.

\section{Middle/Late Miocene Boundary}

The middle/late Miocene boundary has been calibrated to the earliest part of Chron C5 (Berggren et al., 1985). The base of Chron C5 was recorded only at Site $747(\sim 50$ mbsf in Hole 747B) and the (apparent) LO of Neogloboquadrina nympha is associated with the (younger) part of Chron C5.

\section{Miocene/Pliocene Boundary}

The Miocene/Pliocene boundary has recently been calibrated to a level within the early part of the Gilbert Chron at 5 Ma (Zijderveld et al., 1986; Hilgen and Langereis, 1988; Channell et al., 1988; McKenzie et al., 1988). On the Kerguelen Plateau this boundary has been interpreted to lie within an unconformity at Sites 747,748 , and 751 based upon biosiliceous stratigraphy.

\section{Pliocene/Pleistocene Boundary}

The Pliocene/Pleistocene boundary is essentially correlated with the top of the Olduvai Subchron of the Matuyama Chron at $\sim 1.65 \mathrm{Ma}$. This boundary is not associated with any particular event in the Kerguelen Plateau planktonic foraminifer record, but can be correlated approximately on the basis of its biosiliceous elements (see Lazarus et al., this volume; Harwood, this volume).

\section{SPECIES LIST AND TAXONOMIC NOTES}

Taxa identified at Sites 747, 748, and 751 on the Kerguelen Plateau are listed below. Comments clarifying taxonomic usage or other details of distribution or suspected synonymies are made where relevant. Emphasis has been placed upon illustrating taxa of biostratigraphic or biogeographic importance rather than on comprehensive documentation of all taxa present (Plates 1-4).

Globigerina bulloides d'Orbigny, 1839. Plate 3, Fig. 15; Plate 4, Fig. 16.

Globigerina falconensis Blow, 1959. The morphology (and stratigraphic distribution) of forms referred here to $G$. falconensis is similar to $G$. bulloides, save for small, bulla-like final chambers; they may, in fact, be kümmerform $G$. bulloides. Apertural lips were not observed on these forms.

Globigerina praebulloides Blow and Banner, 1963.

Globoturborotalita brazieri Jenkins, 1966. Trilobate, cancellate, spinose form characteristic of and fairly common in the lower Miocene. Plate 1, Figs. 4 and 5.

Globoturborotalita connecta Jenkins, 1964. Compact trilobate, strongly cancellate form characteristic of the lower Miocene and related to the $G$. woodi-G. brazieri group (see Chaproniere, 1988). Plate 1, Figs. 6 and 7.

Globoturborotalita sp. cf. G. labiacrassata Jenkins. A fairly large, robust, cancellate-spinose form with distinct apertural rim that occurs fairly commonly in the lower Miocene of Kerguelen Plateau sites. It differs from typical G. labiacrassata (Oligocene) in its more evolute test and more inflated chambers and lobulate outline. The two forms exhibit a disjunct stratigraphic range as well. We have been unable to place this form among described Austral early Neogene taxa. Plate 1, Figs. 1-3.

Globoturborotalita woodi Jenkins, 1960. Common in the middle-early late Miocene of Kerguelen Plateau sites. It occurs together with $G$. bulloides but more commonly to the (near) exclusion of that taxon. Plate 3, Fig. 16.

Globorotalia conoidea Walters, 1965. This form occurs only rarely and sporadically in the late Miocene on Kerguelen Plateau.

Globorotalia miozea Finlay, 1939. Plate 2, Figs. 15 and 16.

Globorotalia panda Jenkins, 1960. This form mimics the early Pliocene G. margaritae in its arcuate axial profile. It occurs briefly but in high abundance in the middle Miocene of Site 747 following the disappearance of $G$. praescitula, from which it evolved. Plate 3, Figs. 1-3.

Globorotalia praescitula Blow, 1959. Plate 2, Fig. 14.

Globorotalia puncticulata Deshayes, 1832. This taxon occurs fairly frequently in a few samples in the late Pliocene-Pleistocene of Site 747 (see also Shipboard Scientific Party, 1989, pp. 107-109). This record appears quite anomalous in the light of its known distribution (and apparent restriction to the Pliocene) elsewhere (Kennett and Srinivasan, 1983, pp. 104-116). Plate 4, Figs. 10 and 11.

Globorotalia scitula Brady, 1882. Plate 3, Figs. 5-7).

Globorotalia sphericomiozea Walters, 1965. This small, strongly inflated form occurs infrequently in the lower-middle Pliocene of Site 747 , preceding and briefly overlapping $G$. puncticulata. Plate 4, Figs. 12 and 13.

Globorotalia zealandica Hornibrook, 1988. Distinguished from its ancestor Paragloborotalia incognita by axial compression of the test (leading to the typical globorotalid morphology) and a distinctly arching, rimmed aperture. The surface is distinctly pitted (hexagonal pore pits), an ancestral trait inherited from $P$. incognita (see also Cifelli and Scott, 1983), which is reduced in its descendant $G$. praescitula and essentially lost in G. panda. Plate 2, Figs. 12 and 13.

Paragloborotalia incognita Walters, 1965. This form occurs (together with catapsydracids) in high numbers in early Miocene assemblages on the Kerguelen Plateau and would appear to be fairly resistant to dissolution. Plate 2, Figs. 6-8.

Paragloborotalia mayeri Cushman and Ellisor, 1939. Occurs sporadically and in fairly low abundance in the middle Miocene of Kerguelen Plateau. Plate 3, Fig. 4.

Paragloborotalia nana Bolli, 1957.

Paragloborotalia semivera Hornibrook, 1961. Occurs together with, but in subordinate numbers to, $P$. incognita in the early Miocene of Kerguelen Plateau. 
Paragloborotalia sp. cf. P. siakensis LeRoy, 1939.

Tenuitella angustiumbilicata Bolli, 1957.

Tenuitella clemenciae Bermudez, 1961.

Tenuitella minutissima Bolli, 1957.

Tenuitella munda Jenkins, 1966.

Tenuitella pseudoedita Subbotina, 1960.

Globigerinita juvenilis Bolli, 1957.

Globigerinita praestainforthi Blow, 1959. Plate 2, Fig. 1.

Globigerinita uvula Ehrenberg, 1861.

Turborotalita quinqueloba Natland, 1938. Sporadic occurrences (with $N$. pachyderma) in Pleistocene-Pliocene levels on the Kerguelen Plateau. Plate 4, Fig. 17.

Globigerinella calida Parker, 1962. Plate 4, Fig. 15.

Catapsydrax dissimilis Cushman and Bermudez, 1937. The LO of this form was between Subchrons C5D and C5E at Site 747; at a level just above Subchron C5D (terminated by a disconformity) at Site 748; and in Subchron C5E (terminated by dissolution) at Site 751. Plate 1, Figs. 11-16.

Catapsydrax unicavus Bolli, Loeblich and Tappan, 1957. We have tried to maintain a consistent separation between forms with a single (unicavus) and multiple (dissimilis) infralaminal accessory apertures (see also Kennett and Srinivasan, 1983; cf. Bolli and Saunders, 1985, who have grouped these two forms together). In early Miocene dissolution facies $C$. dissimilis, $C$. unicavus, Globorotaloides suteri, and $P$. incognita are essentially the only taxa preserved in some samples. The LO of C. unicavus is in Subchron C5D at Sites 747 and 748; at Site 751 it disappeared in Subchron C5E, approximately $1 \mathrm{~m}$.y. earlier, as a result of strong dissolution at that location. Plate 1, Fig. 10.

Subbotina? sp. cf. angiporoides Hornibrook, 1965. Robust, strongly cancellate forms bearing a resemblance to $S$. angiporoides occur in late Oligocene and early Miocene assemblages. They may, in fact, be referable to Catapsydrax. Plate 1, Fig. 8.

Globorotaloides suteri Bolli, 1957. Plate 1, Fig. 9.

Globorotaloides variabilis Bolli, 1957.

Neogloboquadrina continuosa Blow, 1959. Commonly associated with $N$. nympha in the middle Miocene, this form extends into the late Miocene and early Pliocene and would appear to be the ancestor of $N$. pachyderma. Plate 4, Fig. 7 .

Neogloboquadrina nympha Jenkins, 1966. This is a common and characteristic form of (predominantly) the middle Miocene on the Kerguelen Plateau. Morphologically, it is isomorphic with the low-mid-latitude $N$. acostaensis, from which it differs in its consistently smaller size, in the absence of the thick apertural rim that extends over the umbilical region in some forms, and in the distinctly different stratigraphic range. The type level of $N$. nympha is in the G. mayeri Zone (Waiauan Stage) of New Zealand (equivalent to Zone N14 approximately) but its lower range was said to extend into the Lillburnian Stage (Jenkins, 1971, p. 122), which extends into the lower part of the middle Miocene, equivalent to Zones N9-N13 (see also Edwards et al., 1988) and consistent with the FO of this taxon on the Kerguelen Plateau at a level correlative with MPC 5AD (approximately equivalent to Zone N10 of low latitudes; i.e., in the middle part of the middle Miocene). Jenkins (1971) gave an upper limit for this taxon of Tongaporutuan Stage (= Tortonian; i.e., upper Miocene.) We have been unable to verify the upper limit of this form on the Kerguelen Plateau. Neogloboquadrina nympha appears to have disappeared at about the time of Subchron C $5 \mathrm{~N}$ (early late Miocene), although it may extend to a younger (late Miocene) level at Site 748. Plate 4, Figs. 1-6.

Neogloboquadrina pachyderma Ehrenberg, 1861. The FO of this taxon on the Kerguelen Plateau appears to be in the latest Miocene, at a level correlative with Chron $4(\sim 7 \mathrm{Ma})$ and Subchron 4A ( $>8 \mathrm{Ma})$ at Sites 747 and 751 , respectively. At Site 748 , its initial occurrence is delayed by an upper Miocene-lower Pliocene unconformity. I have not found this form in its typical morphology in middle-early late Miocene assemblages on Kerguelen Plateau, and I remain skeptical of records that attribute a middle Miocene FO to this taxon. It is possible that early records of this taxon refer to $N$. nympha (Jenkins, 1971; see also Jenkins and Srinivasan, 1986). Plate 4, Figs. 8 and 9.

\section{ACKNOWLEDGMENTS}

I wish to thank the master and crew of the JOIDES Resolution for their cooperation and assistance on board ship. Technical assistance was rendered by Marla Poag and Luc Beaufort and the illustrations prepared by Judy Commeau of the U.S.G.S (Woods Hole Branch) on an ETEC scanning electron microscope. Discussions with shipboard colleagues M.-P. Aubry, D. Harwood, D. Lazarus, F. Heider, and H. Inokuchi as well as with K. G. Miller and J. Wright aided in clarifying problems associated with the bio- and magnetostratigraphic interpretations. I would like to thank K. G. Miller, L. D. Stott, and E. Thomas for critical reviews and comments on an early draft of this paper. Part of this work was supported by a grant from JOI-USSAC. This is Woods Hole Oceanographic Institution Contribution Number No. 7566.

\section{REFERENCES}

Berggren, W. A., 1977. Late Neogene planktonic foraminiferal biostratigraphy of the Rio Grande Rise (South America). Mar. Micropaleontol., 3:265-313.

Berggren, W. A., Aubry, M.-P., and Hamilton, N., 1983. Neogene magnetostratigraphy of Deep Sea Drilling Project Site 516 (Rio Grande Rise, South Atlantic). In Barker, P. F., Carlson, R. L., Johnson, D. A., et al., Init. Repts. DSDP, 72: Washington (U.S. Govt. Printing Office), 675-713.

Berggren, W. A., Kent, D. V., and Van Couvering, J. A., 1985. The Neogene: Part 2. Neogene geochronology and chronostratigraphy. In Snelling, N. J. (Ed.), The Chronology of the Geological Record. Geol. Soc. London Mem., 10:211-260.

Berggren, W. A., and Miller, K. G., 1988. Palecgene tropical planktonic foraminiferal biostratigraphy and magnetostratigraphy. Micropaleontology, 34:362-380.

Blow, W. H., 1969. Late middle Eocene to Recent planktonic foraminiferal biostratigraphy. In Brönniman, P., and Renz, H. H. (Eds.), Proc. First Int. Conf. Planktonic Microfossils, Geneva: Leiden (E. J. Brill), 1:199-422.

Bolli, H. M., and Saunders, J. B., 1985. Oligocene to Holocene low latitude planktonic foraminifera. In Bolli, H. M., Saunders, J. B., and Perch-Nielsen, K. (Eds.), Plankton Stratigraphy: Cambridge (Cambridge Univ. Press), 11-16.

Channell, J. T., Rio, D., and Thunnel, R. C., 1988. Miocene/Pliocene boundary magnetostratigraphy at Capo Spartivento, Calabrio, Italy. Geology, 16:1096-1099.

Chaproniere, G.C.H., 1988. Globigerina woodi from the late Oligocene and early Miocene of southeastern Australia. Foraminiferal Res., 18:124-129.

Cifelli, R., and Scott, G. H., 1983. The New Zealand early Miocene globorotaliids Globorotalia incognita Walters and Globorotalia zealandica Hornibrook. J. Foraminiferal Res., 13:163-166.

Edwards, A. R., Hornibrook, N. de B., Raine, J. I., Scott, G. H., Stevens, G. R., Strong, C. P., and Wilson, G. J., 1988. A New Zealand Cretaceous-Cenozoic geological time scale. New Zealand Geological Survey Record, 35:135-149.

Hilgen, F. J., and Langereis, C. G., 1988. The age of the MiocenePliocene boundary in the Capo Rosello area (Sicily). Earth Planet. Sci. Lett., 91:214-222.

Jenkins, D. G., 1966. Planktonic foraminifer zones and new taxa from the Danian to lower Miocene of New Zealand. N. Z. J. Geol. Geophys., 8:1088-1126.

1971. New Zealand Cenozoic planktonic foraminifers. N. Z. Geol. Surv. Paleontol. Bull., 42:1-278.

1975. Cenozoic planktonic foraminiferal biostratigraphy of the Southwestern Pacific and Tasman Sea, DSDP Leg 29. In Kennett, J. P., Houtz, R. E., et al., Init. Repts. DSDP, 29: Washington (U.S. Govt. Printing Office), 449-467.

Jenkins, D. G., and Srinivasan, M. S., 1986. Cenozoic planktonic foraminifers from the equator to the subantarctic of the southwest Pacific. In Kennett, J. P., von der Borch, C. C., et al., Init. Repts. DSDP, 90: Washington (U.S. Govt. Printing Office), 795-834. 
Kennett, J. P., and Srinivasan, M. S., 1983. Neogene Planktonic Foraminifera: A Phylogenetic Atlas: Stroudsburg, PA (Hutchinson Ross).

Loeblich, A. R., Jr., and Tappan, H. E., 1988. Foraminiferal Genera and Their Classification: New York (Van Nostrand-Reinhold).

McKenzie, J. A., Hodell, D. A., Mueller, P. A., and Mueller, D. W., 1988. Application of strontium isotopes to late Miocene-early Pliocene stratigraphy. Geology, 16:1022-1025.

Miller, K. G., Feigenson, M. D., and Wright, J. D., 1991. Miocene isotope standard section, DSDP Site 608: an evaluation of isotope and biostratigraphic resolution. Paleoceanography, 6(1):33-52.

Shipboard Scientific Party, 1989. Site 747. In Schlich, R., Wise, S. W., Jr., et al., 1989. Proc. ODP, Init. Repts., 120: College Station, TX (Ocean Drilling Program), 89-156.
Stott, L. D. and Kennett, J. P. 1990. Antarctic Paleogene planktonic foraminifer biostratigraphy: ODP Leg 113 Site 689 and 690 . In Barker, P. F., Kennett, J. P., et al., Proc. ODP., Init Repts., 113: College Station, TX (Ocean Drilling Program), 549-569.

Zijderveld, J.D.A., Zachariasse, J. W., Verhallen, P.J.J.M., and Hilgen, F. J., 1986. The age of the Miocene/Pliocene boundary. Newsl. Stratigr., 16:169-181.

Date of initial receipt: 5 April 1990

Date of acceptance: 12 December 1990

Ms 120B-153 


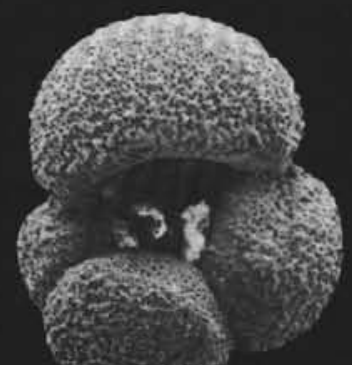

1

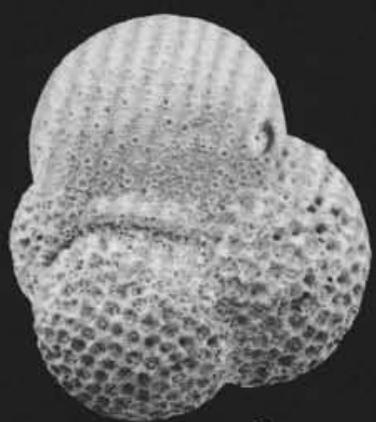

8

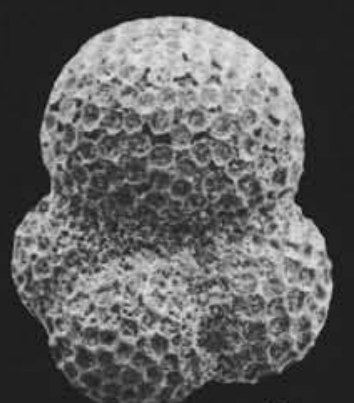

9

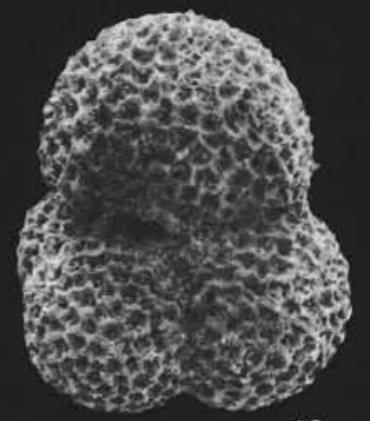

13

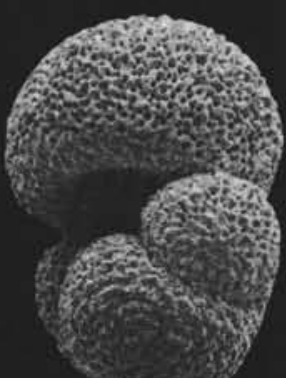

2

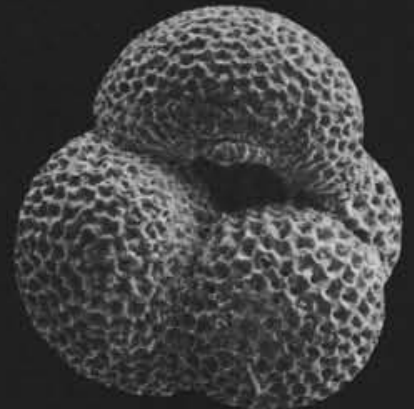

7

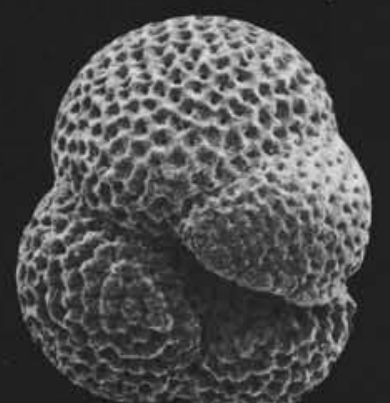

10

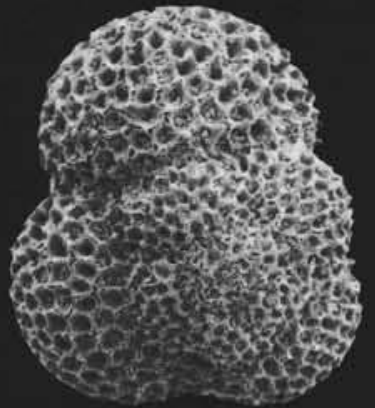

14
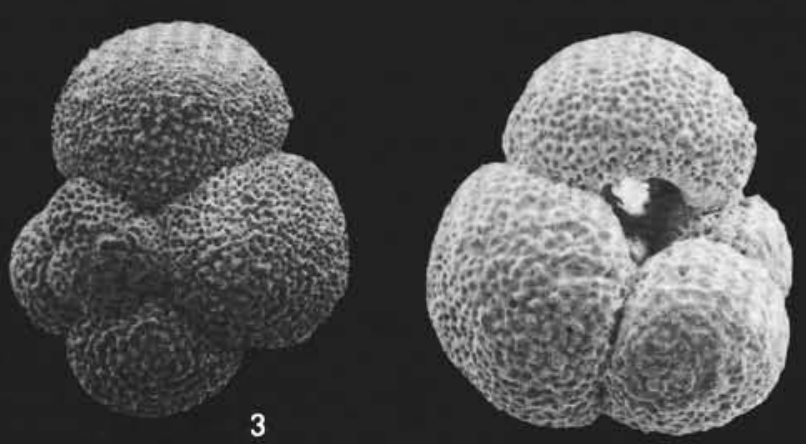

4

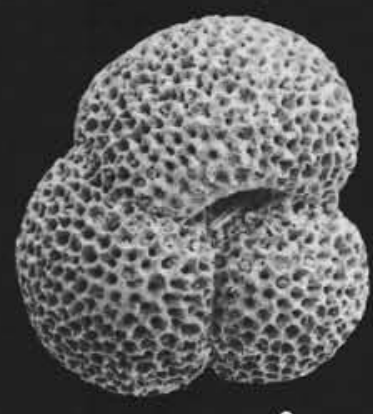

6

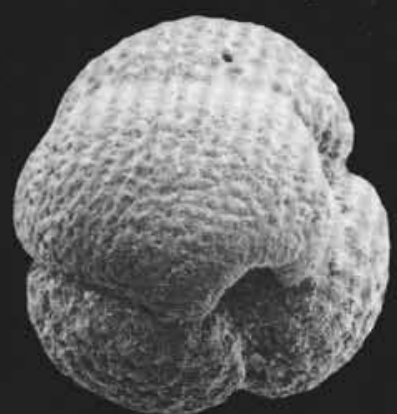

11

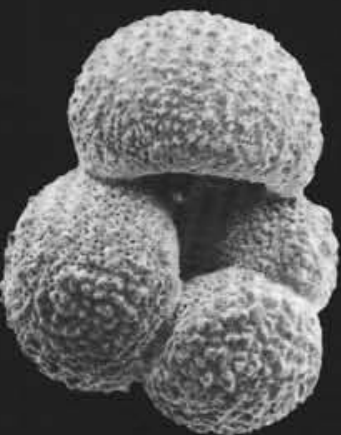

5
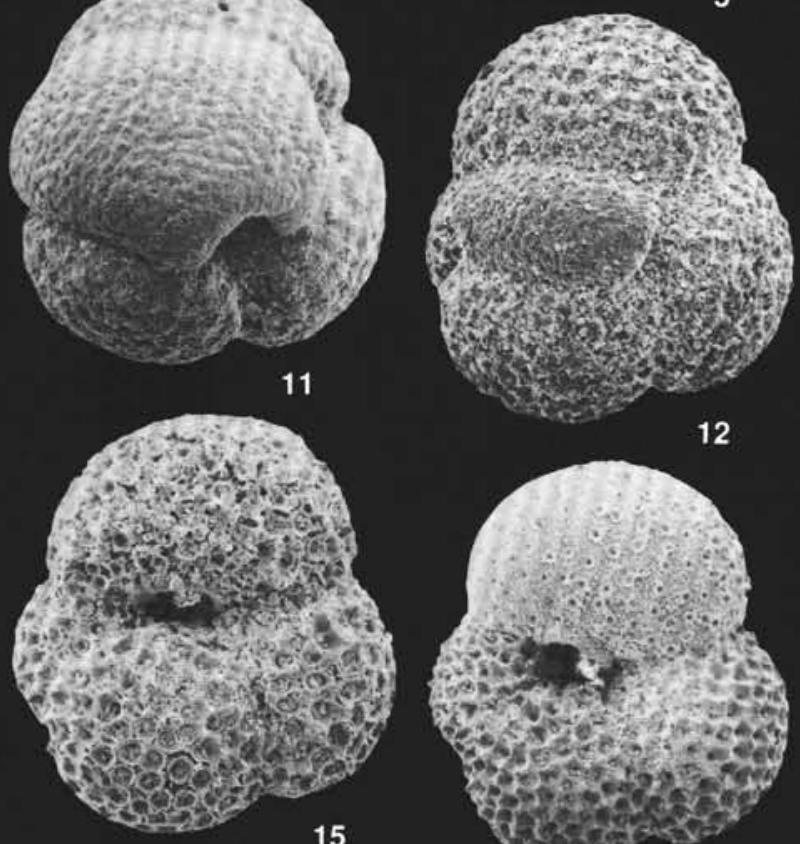

15
12

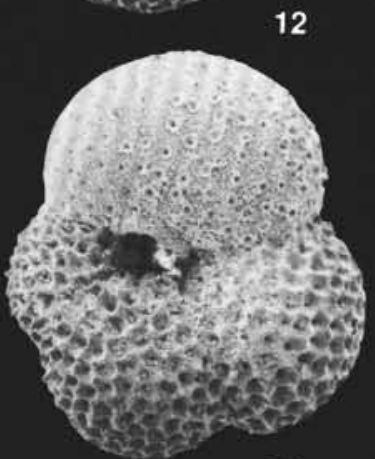

16

Plate 1. Early Miocene. 1-3. Globoturborotalita sp. cf. G. labiacrassata (Jenkins). Sample 120-748B-7H-4, 125-129 cm; (1, 3) $\times 80 ;(2) \times 95.4$, 5. Globoturborotalita brazieri (Jenkins). Sample 120-748B-8H-5, 125-129 cm, $\times 95.6,7$. Globoturborotalita connecta (Jenkins). Sample $120-748 \mathrm{~B}-8 \mathrm{H}-5,125-129 \mathrm{~cm}$; (6) $\times 140$; (7) $\times 120$. 8. Subbotina? sp. cf. S. angiporoides. Sample $120-747 \mathrm{~A}-16 \mathrm{H}-5,40-44 \mathrm{~cm}, \times 85$. 9 . Globorotaloides suteri (Bolli). Sample 120-747A-10H-6, 40-44 cm, $\times 120$. 10. Catapsydrax unicavus (Bolli, Loeblich and Tappan). Sample 120-748B-7H-1, 40-44 cm, $\times 140$. 11. Catapsydrax dissimilis (Cushman and Bermudez). Sample 120-747A-11H-6, 40-44 cm, $\times 110.12-14$. Catapsydrax dissimilis (Cushman and Bermudez). Sample 120-748B-7H-5, 125-129 cm; $(12) \times 120 ;(13) \times 95 ;(14) \times 100 ;(13,14)$ nonbullate forms. 15-16. Catapsydrax dissimilis (Cushman and Bermudez). Sample 120-748B-6H-4, $40-44 \mathrm{~cm}$; $(15) \times 120$; (16) $\times 95$. Nonbullate forms: note differential etching effects of dissolution on nonbullate individuals 13-16. 

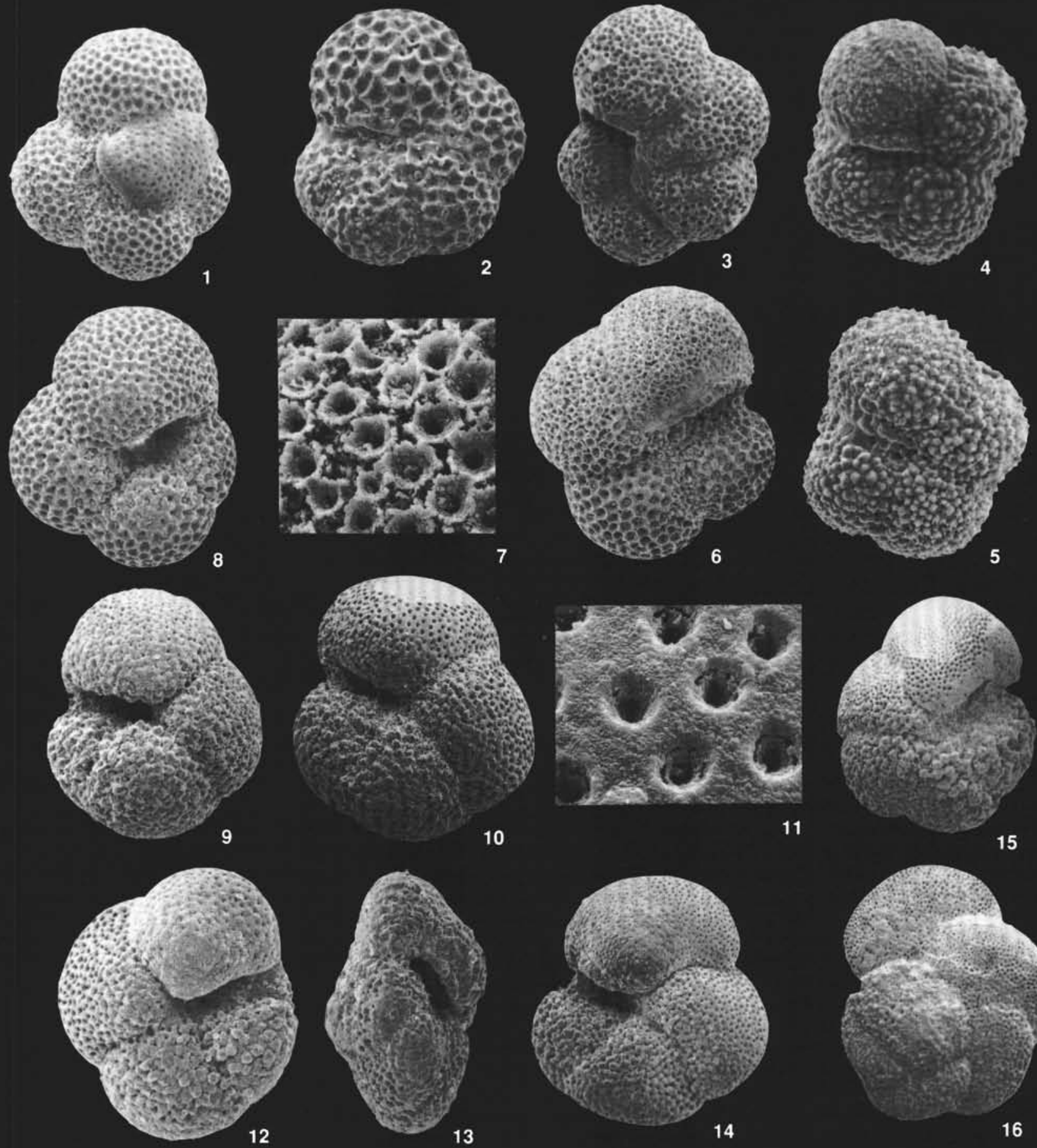

15

Plate 2. Early Miocene. 1. Globigerinita praestainforthi (Blow). Sample 120-747A-14H-6, $40-44 \mathrm{~cm}, \times 185$. 2. Globorotaloides variabilis (Bolli) Sample 120-747A-10H-6, 40-44 cm, $\times 140$. 3. Paragloborotalia $\mathrm{sp}$. cf. P. siakensis (LeRoy). Sample 120-747A-14H-2, 40-44 cm, $\times 155.4,5$. Paragloborotalia nana (Bolli). Sample 120-748B-8H-3, 40-44 cm; (4) $\times 265$; (5) $\times 240$. 6-8. Paragloborotalia incognita (Walters). Sample $120-748 \mathrm{~B}-6 \mathrm{H}-4,40-44 \mathrm{~cm} ;(6)$ and $(8) \times 145 ;$ (7) detail of last chamber of Figure $6, \times 575$. 9-11. Globorotalia zealandica (Hornibrook); (9) Sample 120-747A-9H-6, 40-44 cm; (10) Sample 120-747A-9H-2, 40-44 cm; (10) $\times 105$; (11) Sample 120-747A-9H-2, 40-44 cm, detail of last chamber of Figure 9, $\times 955$. Note absence of spine bases typical of Globorotalia s.s. 12, 13. Globorotalia zealandica Hornibrook. Sample 120-748B-6H-4, $40-44 \mathrm{~cm} ;(12) \times 155$; (13) $\times 135$. 14. Globorotalia praescitula (Blow). Sample 120-748B-6H-4, $40-44 \mathrm{~cm}, \times 115$. 15, 16. Globorotalia miozea (Finlay). Sample 120-747A-9H-6, 40-44 cm; (15) $\times 80 ;(16) \times 70$. 


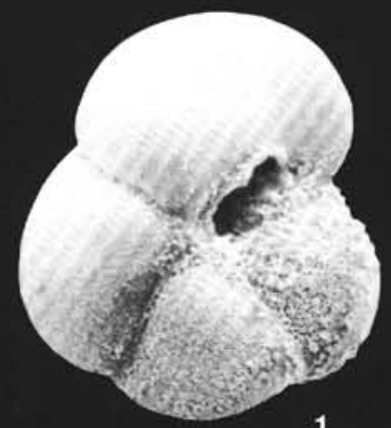

1

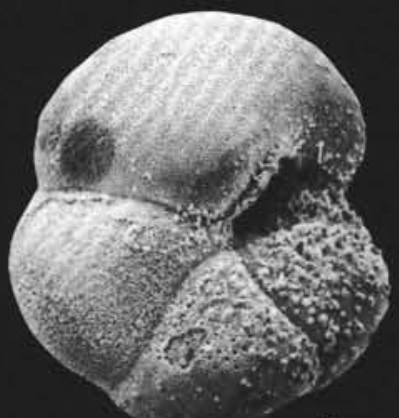

5

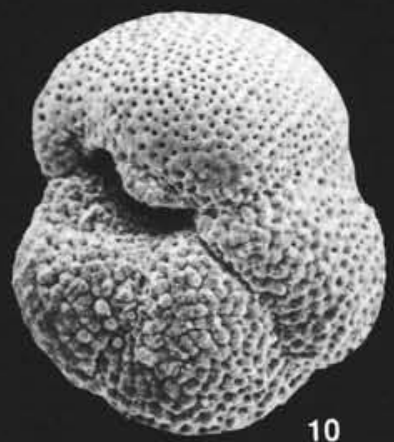

10

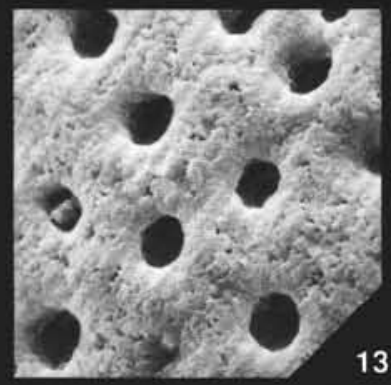

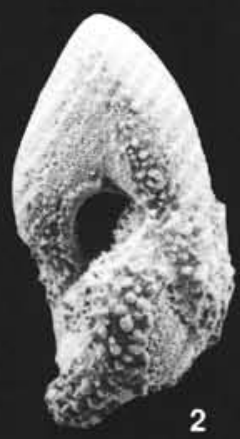
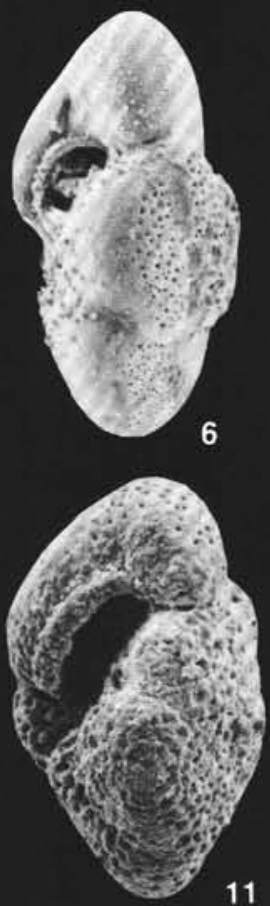

11

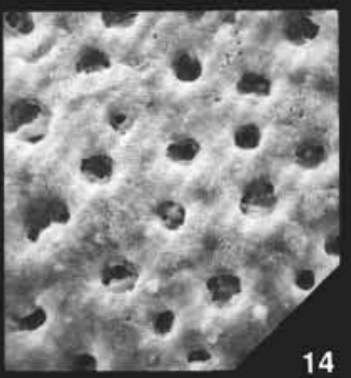

14
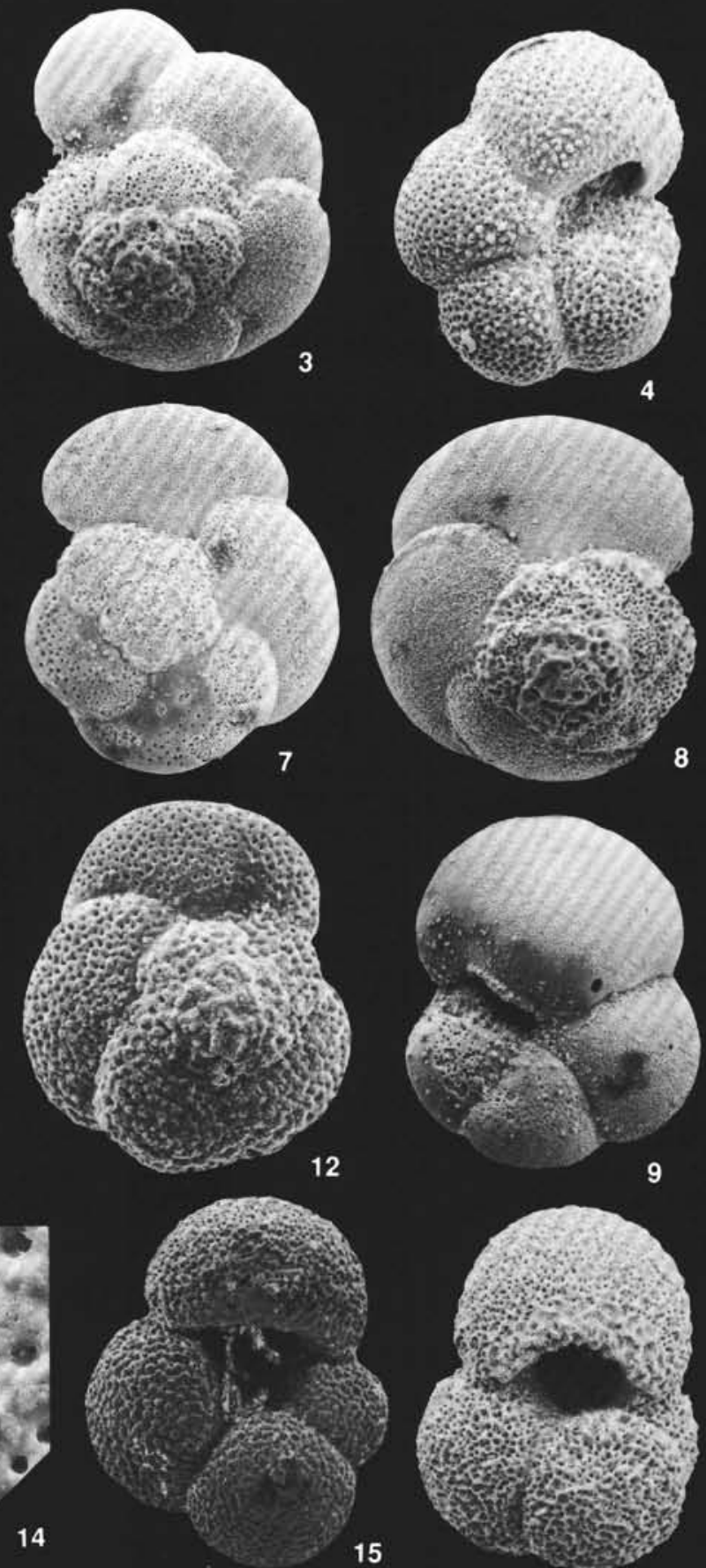

16

Plate 3. Early-middle Miocene. 1-3. Globorotalia panda (Jenkins). Sample 120-747A-7H-6, $40-44 \mathrm{~cm} ;(1) \times 110 ;(2) \times 125 ;(3) \times 95.4$. Paragloborotalia mayeri (Cushman and Ellisor). Sample 120-747A-9H-6, 40-44 cm, $\times 100$. 5-7. Globorotalia scitula (Brady). Sample 120-748B-5H-7, 40-44 cm; (5) $\times 25$; (6) $\times 110$; (7) $\times 105$. 8-9. Globorotalia scitula (Brady). Sample 120-747A-5H-6, 40-44 cm, $\times 105.10-14$. Globorotalia zealandica (Walters). Sample 120-751A-17H-5, 32-36 cm; $(10-12) \times 120 ;(13)$ detail of last chamber in Figure 10, $\times 1240 ;(14)$ detail of last chamber in Figure 12, $\times 810$. 15. Globigerina bulloides (d'Orbigny). Sample 120-748B-6H-1, 80-84 cm, $\times 100$. 16. Globoturborotalita woodi (Jenkins). Sample 120-747A-6H-2, 40-44 cm, $\times 100$. 


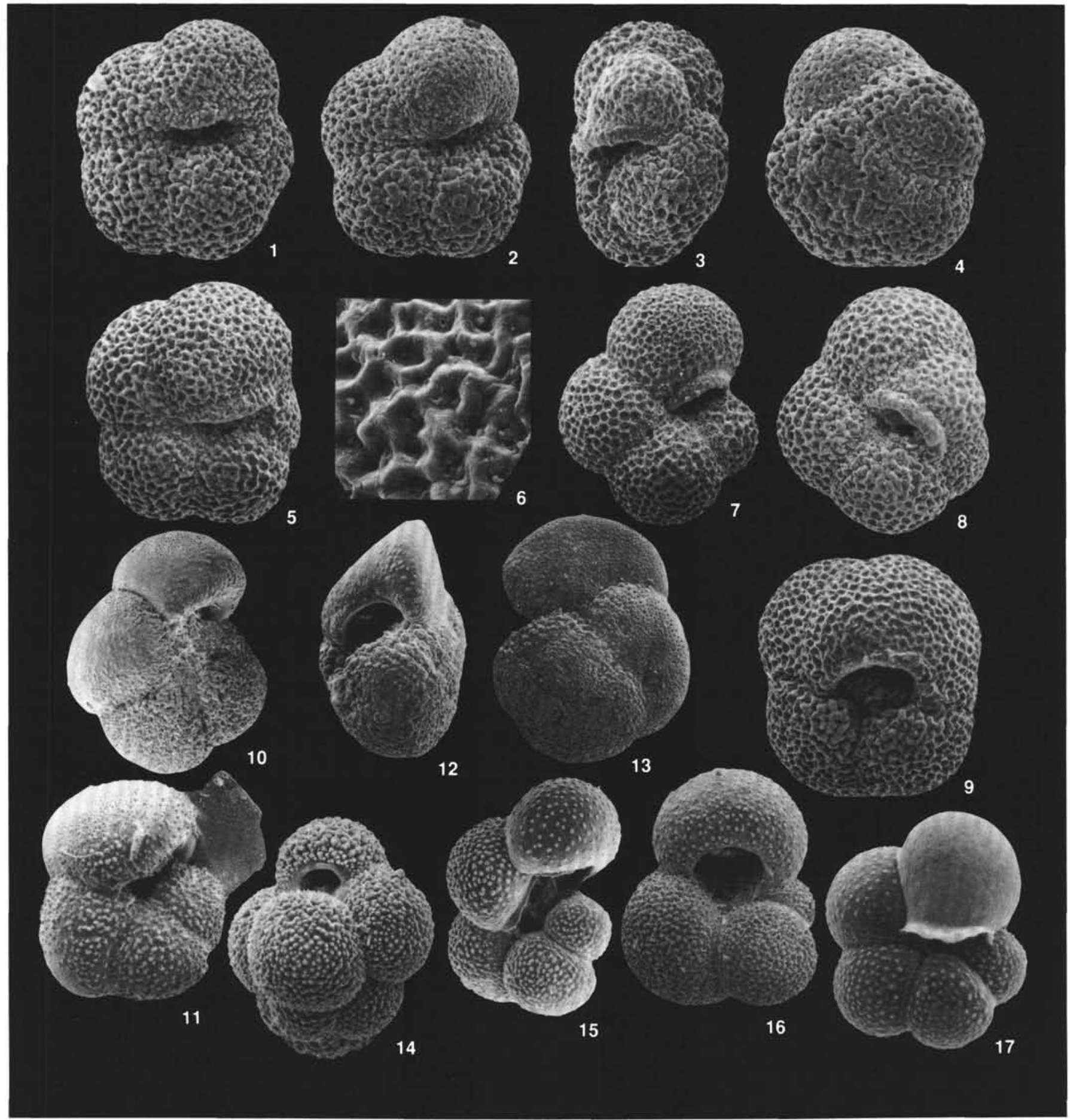

Plate 4. Late Neogene (middle Miocene to Pleistocene). 1-4. Neogloboquadrina nympha (Jenkins). Sample 120-748B-5H-7, 40-44 cm, $\times 175.5$, 6. Neogloboquadrina nympha (Jenkins). Sample 120-747A-4H-4, 40-44 cm. (5) $\times 170$; (6) detail of last chamber in Figure 5 , $\times 710$. No evidence of spine base at junction of interpore ridges. 7. Neogloboquadrina continuosa (Blow). Sample 120-747A-4H-CC, $\times 135$. 8. Neogloboquadrina pachyderma (Ehrenberg). Sample 120-747A-4H-4, 40-44 cm, $\times 155$, early form. 9. Neogloboquadrina pachyderma (Ehrenberg). Sample 120-747A-1H-2, 40-44 cm, $\times 115$, typical form. 10, 11. Globorotalia puncticulata (d'Orbigny). Sample $120-747 \mathrm{~A}-1 \mathrm{H}-\mathrm{CC}$. $(10) \times 55 ;(11) \times 80$. 12, 13. Globorotalia sphericomiozea (Walters). Sample $120-747$ A-2H-CC. $(12) \times 95 ;(13) \times 70$. 14. Globigerinita uvula (Ehrenberg). Sample 120-747A-1H-2, 40-44 cm, $\times 245$. 15. Globigerinella calida (Parker). Sample 120-747A-2H-4, 40-44 cm, $\times 140$. 16. Globigerina bulloides (d'Orbigny). Sample 120-747A-1H-CC, $\times 90$. 17. Turborotalita quinqueloba (Natland). Sample 120-747A-1H-2, 40-44 cm, $\times 200$. 